\title{
TIA1 variant drives myodegeneration in multisystem proteinopathy with SQSTM1 mutations
}

\author{
YouJin Lee, ${ }^{1}$ Per Harald Jonson, ${ }^{2}$ Jaakko Sarparanta, ${ }^{2}$ Johanna Palmio, ${ }^{3}$ Mohona Sarkar, ${ }^{4}$ Anna Vihola, ${ }^{2}$ Anni Evilä, ${ }^{2}$ \\ Tiina Suominen, ${ }^{3}$ Sini Penttilä, ${ }^{3}$ Marco Savarese, ${ }^{2}$ Mridul Johari, ${ }^{2}$ Marie-Christine Minot, ${ }^{5}$ David Hilton-Jones, ${ }^{6}$ Paul Maddison, ${ }^{7}$ \\ Patrick Chinnery, ${ }^{8,9}$ Jens Reimann, ${ }^{10}$ Cornelia Kornblum, ${ }^{10,11}$ Torsten Kraya, ${ }^{12}$ Stephan Zierz, ${ }^{12}$ Carolyn Sue, ${ }^{13}$ Hans Coebel, ${ }^{14}$ \\ Asim Azfer, ${ }^{15}$ Stuart H. Ralston, ${ }^{15}$ Peter Hackman, ${ }^{2}$ Robert C. Bucelli, ${ }^{1}$ J. Paul Taylor, ${ }^{4,16}$ Conrad C. Weihl, ${ }^{1}$ and Bjarne Udd ${ }^{2,3,17}$ \\ 'Department of Neurology, Hope Center for Neurological Diseases, Washington University School of Medicine, St. Louis, Missouri, USA. ${ }^{2}$ Folkhälsan Institute of Cenetics and Department of Medical Genetics, \\ Haartman Institute, University of Helsinki, Helsinki, Finland. ${ }^{3}$ Neuromuscular Research Center, Tampere University Hospital and University of Tampere, Tampere, Finland. ${ }^{4}$ Department of Cell and Molecular \\ Biology, St. Jude Children's Research Hospital, Memphis, Tennessee, USA. ${ }^{5}$ Neuromuscular Competence Center, Centre Hospitalier Universitaire (CHU) de Rennes, Rennes, France. ${ }^{6}$ Nuffield Department of Clinical \\ Neurosciences, University of Oxford, Oxford, United Kingdom. 'Department of Neurology, University of Nottingham, Nottingham, United Kingdom. ${ }^{8}$ MRC-Mitochondrial Biology Unit, University of Cambridge, \\ Cambridge, United Kingdom. ${ }^{9}$ Department of Clinical Neurosciences, Cambridge Biomedical Campus, University of Cambridge, Cambridge, United Kingdom. ${ }^{10}$ Department of Neurology, University Hospital of \\ Bonn, Bonn, Germany. "'Centre for Rare Diseases Bonn (ZSEB), Department of Neurology, University Hospital of Bonn, Bonn, Cermany. ${ }^{12}$ Department of Neurology, Martin-Luther-Universität Halle-Wittenberg, \\ Halle/Saale, Germany. ${ }^{13}$ Department of Neurogenetics, Kolling Institute of Medical Research, Royal North Shore Hospital and University of Sydney, St Leonard's, New South Wales, Australia. ${ }^{14}$ Department of \\ Neuropathology, Charité - Universitätsmedizin Berlin, Berlin, Germany. ${ }^{15}$ Centre for Genomic and Experimental Medicine, MRC Institute of Genetics and Molecular Medicine, University of Edinburgh, Western \\ General Hospital Edinburgh, United Kingdom. ${ }^{16}$ Howard Hughes Medical Institute, Chevy Chase, Maryland, USA. ${ }^{17}$ Department of Neurology, Vaasa Central Hospital, Vaasa, Finland.
}

\begin{abstract}
Multisystem proteinopathy (MSP) involves disturbances of stress granule (SC) dynamics and autophagic protein degradation that underlie the pathogenesis of a spectrum of degenerative diseases that affect muscle, brain, and bone. Specifically, identical mutations in the autophagic adaptor SOSTM1 can cause varied penetrance of 4 distinct phenotypes: amyotrophic lateral sclerosis (ALS), frontotemporal dementia, Paget's disease of the bone, and distal myopathy. It has been hypothesized that clinical pleiotropy relates to additional genetic determinants, but thus far, evidence has been lacking. Here, we provide evidence that a TIA1 (p.N357S) variant dictates a myodegenerative phenotype when inherited, along with a pathogenic SOSTM1 mutation. Experimentally, the TIA1-N357S variant significantly enhances liquid-liquid-phase separation in vitro and impairs SC dynamics in living cells. Depletion of SQSTM1 or the introduction of a mutant version of SQSTM1 similarly impairs SC dynamics. TIA1-N357S-persistent SCs have increased association with SQSTM1, accumulation of ubiquitin conjugates, and additional aggregated proteins. Synergistic expression of the TIA1-N357S variant and a SQSTM1-A390X mutation in myoblasts leads to impaired SC clearance and myotoxicity relative to control myoblasts. These findings demonstrate a pathogenic connection between SC homeostasis and ubiquitin-mediated autophagic degradation that drives the penetrance of an MSP phenotype.
\end{abstract}

\section{Introduction}

Pathogenic mutations in some genes lead to a spectrum of variably penetrant phenotypes that span different organs and postmitotic tissue (1-4). For example, the same mutation in the autophagic adaptor protein SQSTM1 (also known as p62) can cause Paget's disease of the bone (PDB), rimmed vacuolar inclusion body myopathy (RV-IBM), amyotrophic lateral sclerosis (ALS), or frontotemporal dementia (FTD) (1). The term multisystem proteinopathy (MSP) has become useful to describe this growing family of genetic diseases that so far have been reported to have dominant mutations in the pleotropic genes VCP, HNRNPA2B1, HNRN$P A 1$, and SQSTM1 (1-4). Other disease-associated genes with vari-

Authorship note: C.C. Weihl and B. Udd contributed equally to this work. Conflict of interest: J.P. Taylor is a consultant for Inception Sciences. Submitted: August 29, 2017; Accepted: January 9, 2018.

Reference information: / Clin Invest. 2018;128(3):1164-1177.

https://doi.org/10.1172/JCI97103. ably penetrant phenotypic expression of RV-IBM, ALS, and FTD, yet no association with PDB, include TIA1 and MATR3 (5-7). One distinctive feature of the MSP pedigrees is that patients with the same mutation, and even the same mutation within a family, can manifest different phenotypes (i.e., PDB in 1 sibling and ALS in another sibling). MSP also unifies 2 key pathologic features in affected tissue: ubiquitinated aggregates and the accumulation of RNA-binding proteins with low-complexity sequence domains (LCDs) such as TDP-43 (4).

Mutations in several proteins that facilitate ubiquitin-dependent autophagy such as VCP, SQSTM1, UBQLN2, and OPTN are associated with PDB, RV-IBM, ALS, and FTD (1, 2, 8, 9). Disease mutations in these proteins impair the degradation and clearance of ubiquitinated inclusions, resulting in their accumulation. SQSTM1 is an autophagic adaptor protein with a UBA domain and an autophagosome-interacting motif (10). Most pathogenic variants in SQSTM1 are missense mutations within or truncations of the UBA domain (11). These mutations affect the oligomeriza- 
tion of SQSTM1 and its ability to recruit ubiquitinated aggregates to the autophagosome, suggesting that the pathogenesis of MSP and its related diseases are due in part to alterations in protein homeostasis and particularly to autophagic degradation of ubiquitinated proteins in vulnerable tissues (11). One disease-associated SQSTM1 mutation that has been demonstrated to cause the full spectrum of MSP phenotypes (PDB, RV-IBM, ALS, and FTD) is a proline-to-leucine mutation at residue 392 (P392L) in the UBA domain (12-15). This single mutation is the most common genetic cause of PDB but is incompletely penetrant, suggesting that other genetic or environmental factors are needed for the phenotypic manifestation of PDB, RV-IBM, ALS, or FTD (16).

One distinctive pathologic feature seen in MSP-affected tissues is the accumulation of cytoplasmic inclusions of RNA-binding proteins such as TDP-43 (4). Indeed, mutations in RNA-binding proteins with LCDs, including TARDBP, FUS, HNRNPA2B1, HNRNPA1, TIA1, and HNRPDL, cause dominantly inherited forms of MSP, ALS, FTD, and RV-IBM (3, 6, 7, 17-19). These RNA-binding proteins promote the assembly of membrane-less organelles such as stress granules (SGs) through the biophysical process of liquid-liquid phase separation (LLPS) that is mediated via the LCD (20). Importantly, disease-causing mutations in the LCD of these RNA-binding proteins alter their biophysical properties, increase their propensity to undergo LLPS, and result in the accumulation of poorly dynamic SGs that are believed to underlie cellular dysfunction and evolve into the pathological inclusions characteristic of these diseases $(7,20,21)$. Notably, TIA1 is a key component of cytosolic SGs (7). Dominantly inherited mutations within the LCD of TIA1 cause MSP or more discreet clinical manifestations of either ALS-FTD or a distinctive form of distal myopathy termed Welander distal myopathy (WDM) $(6,7)$. As with hnRNPA1, hnRNPA2B1, and TDP-43, disease mutations in the LCD of TIA1 alter its biophysical properties, promote LLPS, enhance fibrillization, and impair SG clearance as a mechanism of their pathogenicity $(6,7)$.

RV-IBMs are defined by a core set of pathologic features including rimmed vacuoles (RVs) and inclusion bodies, seen in degenerative myopathies (22). RVs are present within some myofibers and contain autophagic and membranous debris, such as SQSTM1 and MAP1LC3. Many proteins have been observed to accumulate as inclusions in RV-IBM tissue. These proteins include ubiquitin, $\beta$-amyloid, and TDP-43, with TDP-43 aggregation being specific for RV-IBMs (22). In many cases, protein inclusions and vacuolar debris are stained by Congo red and show birefringence, suggesting that the inclusions contain amyloidogenic proteins. A number of studies now establish that SQSTM1 and/or TDP-43 within myofibers serve as markers of muscle degeneration in RV-IBMs (23-26).

The relationship between SG homeostasis and autophagic protein degradation has been demonstrated in cell cultures $(27,28)$. Specifically, autophagy participates in SG clearance. Autophagymediated SG clearance may also require the ubiquitin segregase $\operatorname{VCP}(27,28)$. Autosomally dominantly inherited mutations in VCP cause MSP, but whether the pathogenesis of MSP is mediated via its effect on SG homeostasis is not known (2). The present study identifies a rare variant in TIA1 that dictates the tissue specificity associated with SQSTM1 mutations. Digenic inheritance of a TIA1-N357S variant with a pathogenic SQSTM1 mutation causes a distal myopathy with RV-IBM pathology. This finding connects SG homeostasis with ubiquitin-dependent autophagic degradation as a key mediator of the phenotypic outcome within the spectrum of RV-IBM, ALS, and FTD and shows the relevance of oligogenic mechanisms as one cause of neurodegenerative-neuromuscular disease.

\section{Results}

Digenic inheritance of an MSP-associated SQSTM1 mutation with a rare TIA1-N357S variant occurs in distal myopathy patients with $R V$ pathology. We have previously described 2 unrelated families with distal myopathy and RV-IBM pathology caused by a c.1165+1 G>A splice donor variant in SQSTM1 (1). This variant generates a truncated SQSTM1 protein that lacks its UBA domain and has been identified in patients with PDB and ALS $(12,13)$. Subsequent to this discovery, we identified an additional 3 patients with distal myopathy and a SQSTM1 p.P392L variant (15). Likewise, this SQSTM1 p.P392L variant has been associated with dominantly inherited PDB, ALS, and FTD, phenotypes that were not present in our patients (12-14). Interestingly, these 3 patients had been previously identified in our cohort of distal myopathy patients carrying a rare TIA1 c.1070A>G; p.N357S; rs116621885 variant with a minor allele frequency (MAF) of 0.007. Surprisingly, our 3 previously reported SQSTM1 c. $1165+1 \mathrm{G}>$ A patients also carried the same rare TIA1 p.N357S variant. Dominant mutations in TIA1 are associated with WDM, a distal myopathy with RV-IBM pathology and, more recently, with ALS and FTD $(6,7)$. Notably, like with the WDM- and ALS-FTD-associated TIA1 mutations, the TIA1 p.N357S variant was present within the LCD at conserved residues (Figure 1A).

We used 2 approaches to identify additional myopathy patients with SQSTM1 mutations and the TIA1 p.N357S variant. First, we analyzed our neuromuscular disease gene panel sequencing results that included SQSTM1 and TIA1 for 1,294 patients with a presumed hereditary muscle disease (15). In addition, we performed Sanger sequencing of SQSTM1 in 14 patients with undetermined distal myopathy and a WDM phenotype, whom we had previously identified as having the TIA1 p.N357S variant. These approaches identified a total of 8 patients from 6 families with the common SQSTM1 p.P392L mutation, 7 of whom also carried the TIA1 p.N357S variant. Notably, none of them had PDB, and all had distal myopathy with RV-IBM pathology, with the exception of 1 patient, who carried only the SQSTM1 p.P392L mutation without the TIA1 p.N357S variant. This patient had a proximal phenotype, without RV-IBM muscle pathology, that had been characterized as limb-girdle muscular dystrophy.

We also identified 3 patients from 2 families with a previously reported SQSTM1 p.M404V mutation that was associated with PDB and dementia $(29,30)$. These patients also carried the TIA1 p.N357S variant and had late-onset, distal predominant myopathy with RV-IBM pathology and no evidence of PDB. One additional patient with distal myopathy and RV-IBM pathology carried the TIA1 p.N357S variant and a rare synonymous SQSTM1 variant, c.1083C > T, p.S361S, which was previously identified in a patient with early-onset dementia (30). This synonymous mutation has a MAF of 0.00008 and is predicted to be disease causing.

MRI of patients' lower extremities revealed extensive fatty degenerative changes in distal muscles, evidenced by focal involvement of lower leg gastrocnemius and soleus muscles (Figure 1C). Muscle biopsies from a patient showed variation in fiber 
A

\begin{tabular}{l|c|c|c|c|}
\hline RRM1 & RRM2 & RRM3 & LCD \\
\hline \multicolumn{4}{c}{357} \\
Human & ...APWMGPNYGVQPPQGQNGSMLPNQPSGYRVAGYETQ \\
Mouse & ...APWMGPNYSVPPPQGQNGSMLPSQPAGYRVAGYETQ \\
Chimp & ...APWMGPNYGVQPPQGQNGSMLPNQPSGYRVAGYETQ \\
Bovine & ...PPWMGPNYGVQPPPGQNGSMMPNQPAGYRVAGFF--
\end{tabular}

B

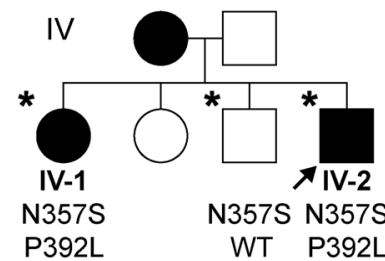

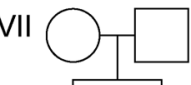

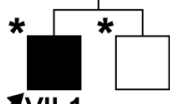

N357S WT

P392L WT

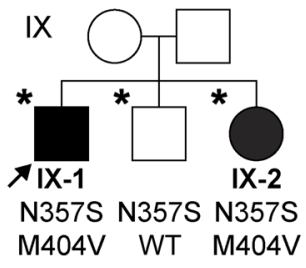

C
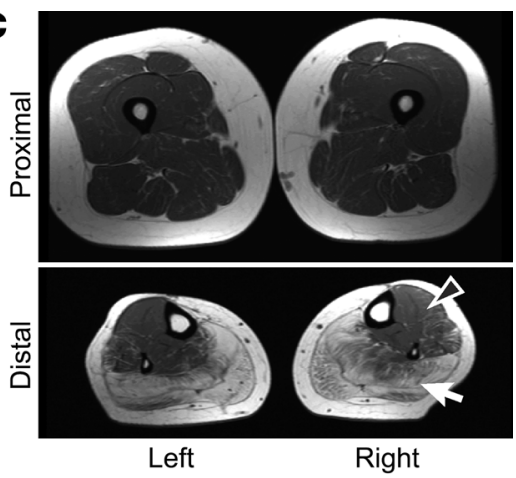

D

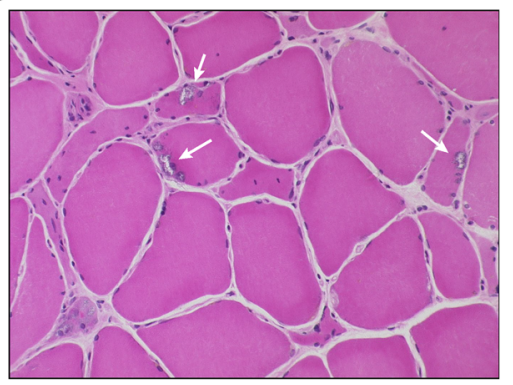

$\mathbf{E}$

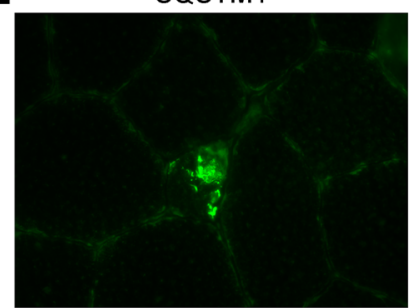

TDP-43

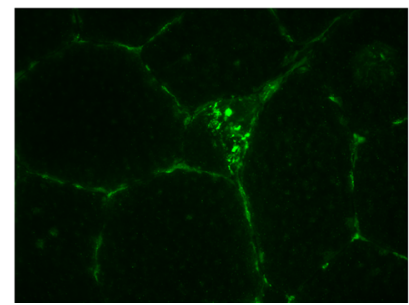

\section{(1)}

TIA1

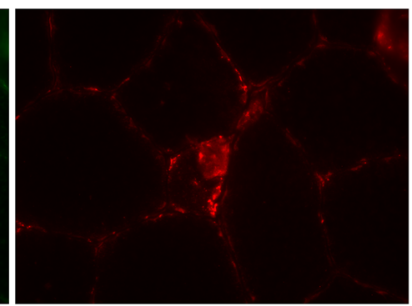

TIA1

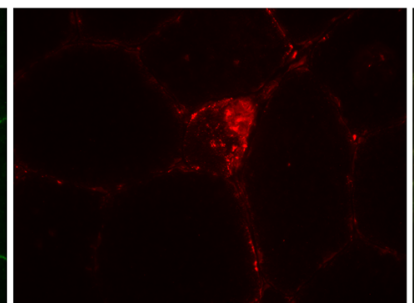

SQSTM1 / TIA1

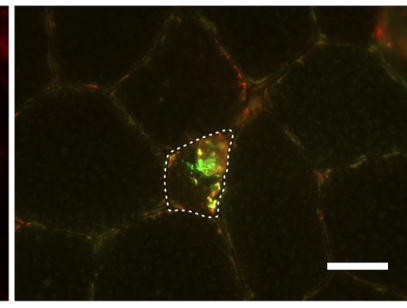

TDP-43/ TIA1

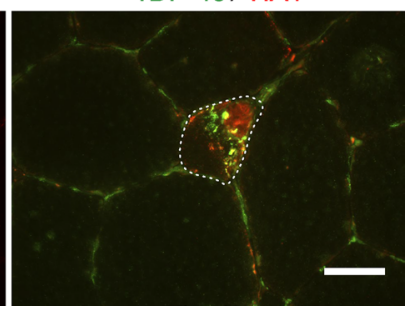

Figure 1. Digenic inheritance of SOSTM1 and TIA1 variants leads to distal myopathy with RV-IBM pathology. (A) Linear diagram of the TIA1 protein highlighting conserved regions of the LCD. Distal myopathy-associated variant positions are shown in yellow and ALS and FTD variants in blue. (B) Pedigrees of families IV, VII, and IX showing segregation. DNA was only available for the patients indicated with an asterisk. (C) Muscle imaging findings for patient $\mathrm{V}-2$ at age 54 years. Severe involvement of all calf muscles was seen on MRI T1-weighted images. The solid white arrow indicates normal muscle, and the arrowhead indicates atrophic muscle with fatty replacement. (D) H\&E staining of a muscle biopsy of the right tibialis from patient XII-1 showing several fibers with RVs (arrows). Original magnification, $50 \mu \mathrm{m}$. (E) Immunofluorescence staining of TIA1 (red) with SQSTM1 (green in upper panel) or TDP-43 (green in lower panel) revealed accumulation and partial colocalization of these proteins in the muscle biopsy from patient $\mathrm{V}-2$. Both sets of images show a RV fiber. The dotted lines denote affected fiber. Scale bars: $50 \mu \mathrm{m}$.

size and RVs consistent with RV-IBM (Figure 1D and Supplemental Figure 1B; supplemental material available online with this article; https://doi.org/10.1172/JCI97103DS1). In pathologically normal muscle, SQSTM1 is diffusely sarcoplasmic, and TDP-43 and TIA1 are myonuclear (Supplemental Figure 1A). In contrast, SQSTM1 and TIA1 accumulated in the patient's muscle sarcoplasm and had some colocalization at RVs as determined by IHC (Figure 1E). In addition, TIA1 accumulated with other RNA-binding proteins such as TDP-43 and HNRNPA2B1 (Figure 1E and Supplemental Figure 1C). Western blot (WB) analysis of skeletal muscle from 1 patient showed normal steady-state levels for SQSTM1 and TIA1 (Supplemental Figure 1D). Table 1 lists the clinical and laboratory characteristics for all SQSTM1-TIA1 patients included in this study. Digenic inheritance was found in 3 families, in which only examined affected family members carried both a SQSTM1
p.P392L or a SQSTM1 p.M404V mutation and a TIA1 p.N357S variant, whereas family members carrying only a TIA1 p.N357S variant were unaffected (Figure 1B).

Importantly, sequencing of 50 patients (26 male, 24 female; average age, $73.7 \pm 7.3$ years) with previously reported pathogenic SQSTM1 mutations, including 40 patients with a SQSTM1-P392L mutation and 2 with a SQSTM1-M404V mutation manifesting with PDB but no muscle weakness, did not reveal any TIA1-N357S variant, supporting the idea that both variants are necessary for a muscle phenotype with RV-IBM pathology (Supplemental Table 1) (31).

TIA1 N357S variant is overrepresented in distal myopathy patients. Although patients in these small pedigrees with only the TIA1 p.N357S variant had no evidence of weakness, we reasoned that the rare TIA1 p.N357S variant may itself be enriched in patients with a distal myopathy phenotype. Indeed, in our large sequencing proj- 
Table 1. Clinical characteristics of the patients

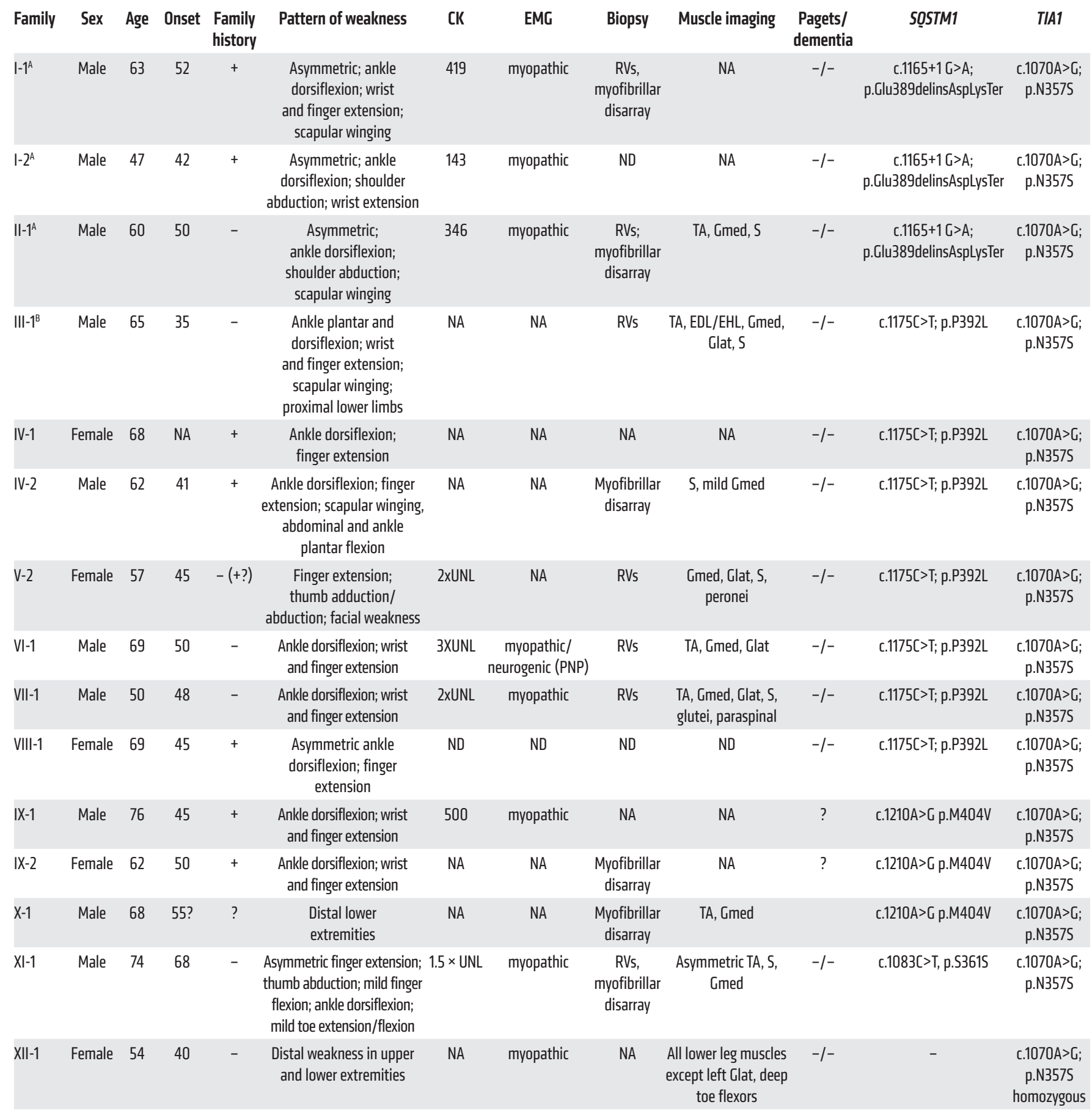

EDL/EHL, long toe extensors; Gmed, gastrocnemius medialis; Glat, gastrocnemius lateralis; NA, not available; ND, not done; PNP, polyneuropathy; S, soleus; TA, tibialis anterior; UNL, upper normal limit. Previously reported patients from ${ }^{A}$ ref. 1 and ${ }^{B}$ ref. 15.

ect involving 1,293 patients with presumed hereditary myopathy, 41 patients (3\%) had the N357S variant (allele frequency 0.0162 vs. 0.007 in the general population), and when this was stratified to those with a distal myopathy phenotype involving upper limbs as well, 17 of 86 (20\%) of these patients carried the variant, including 1 patient who was homozygous for the TIA1 p.N357S variant, making the allele frequency 0.1 for distal myopathy. A similar overrepresentation of this TIA1 p.N357S variant was seen in 5 patients from a second cohort of 51 patients with undiagnosed myopathy revealed by whole-exome sequencing. All 5 patients had a distal predominant phenotype with RV-IBM pathology, and 2 of these patients had an additional c. $1165+1 \mathrm{G}>$ A splice donor variant in SQSTM1 (1). 
A

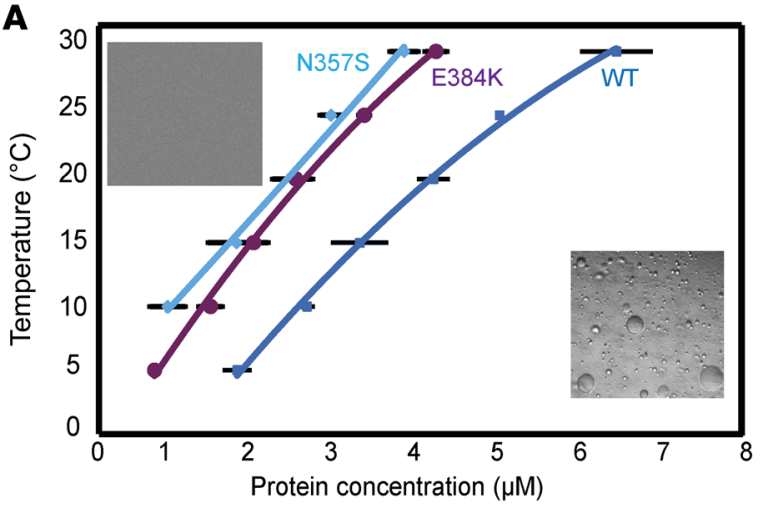

C
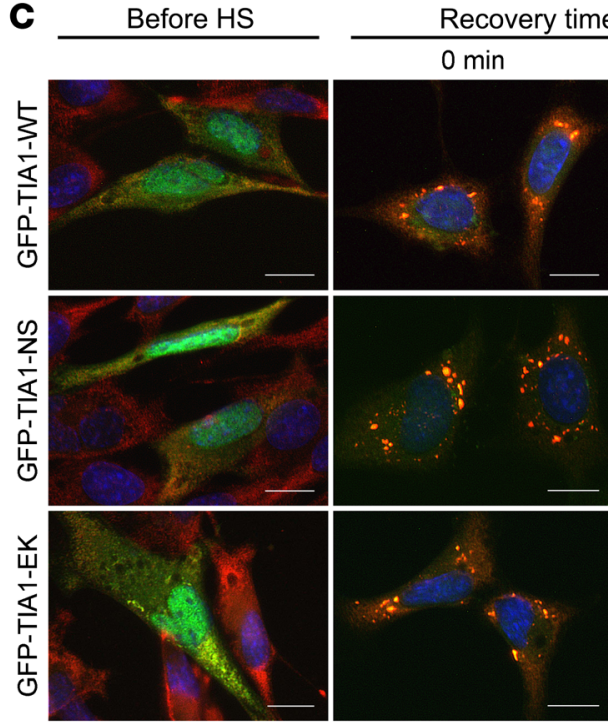

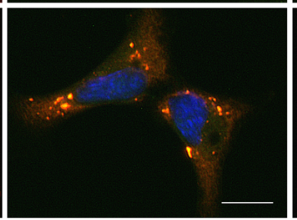

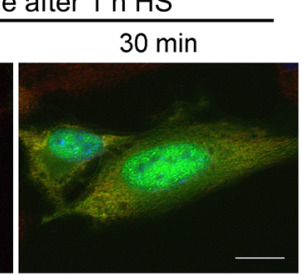

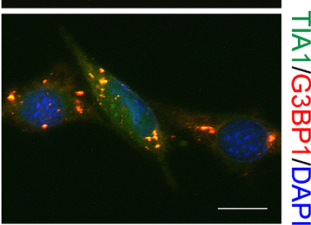

B

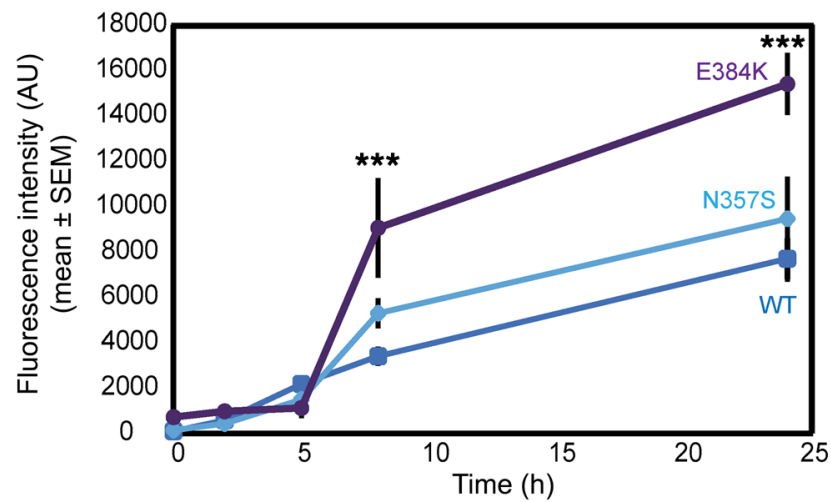

D

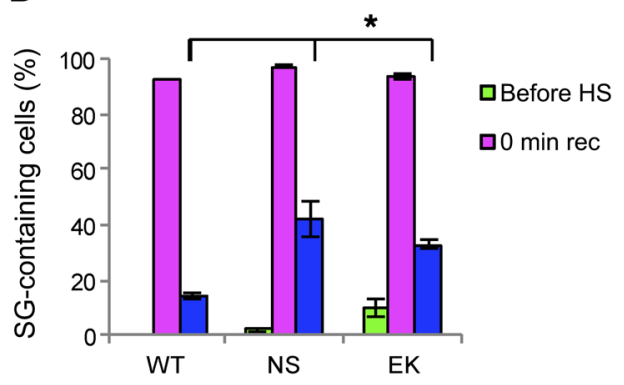

$\mathbf{E}$

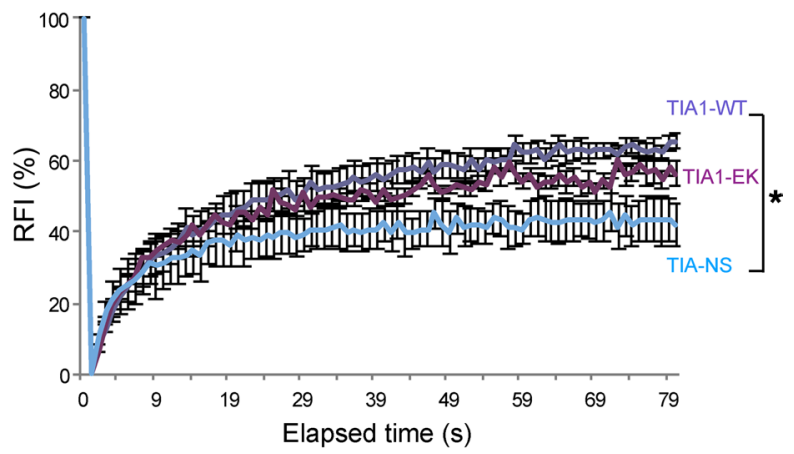

Figure 2. TIA1-N357S variant promotes LLPS and disrupts SC dynamics. (A) Phase diagram of TIA1-WT, -EK, and -NS mapped at physiological conditions. The mean concentration of the light phase (protein depleted phase) and SE are plotted. A quadratic equation was used to fit the trendlines $\left(R^{2}, \mathrm{WT}\right.$, and NS $=0.99$, EK $=0.98 ; P<0.003$ for NS vs. WT and $P<0.0002$ for EK vs. WT, by $\chi^{2}$ test). Insets show characteristic DIC images of light, diffused phase, and dense phase droplets, respectively. (B) Thioflavin T fluorescence intensity of amyloid fibrils at 2.5 $\mu$ M TIA1-WT, -EK, and -NS variants at the indicated time points. The spectrum of BSA (nonamyloid fibril-forming) was measured as a baseline. That baseline was subtracted from all the WT, EK and NS spectra at each time point. ${ }^{* *} P<0.001$, by 2 -way ANOVA with Tukey's multiple comparisons test (NS, $\left.P>0.1\right)$. (C) IF images of MEFs expressing GFP-TIA1-WT, -NS, or -EK immunostained with anti-C3BP1 (red) prior to 1 hour $\mathrm{HS}$ at $42^{\circ} \mathrm{C}$, immediately after HS, or following a 30-minute HS recovery at $37^{\circ} \mathrm{C}$. DAPI nuclear staining is shown in blue. Scale bars: $5 \mu \mathrm{m}$. (D) Bar graph of the percentage of cells containing TIA1/ G3BP1-positive SGs under the conditions described in C. Individual transfected cells were counted and are indicated as the total number of cells. Representative data were pooled from 3 independent experiments $(n=150 \sim 200)$. rec, recovery. (E) Graphical representation of the average relative fluorescence intensity (RFI) following photobleaching of individual SGs from MEFs expressing GFP-TIA1-WT, -NS, or -EK and treated for 1 hour with $0.5 \mathrm{mM}$ arsenite. Representative data were pooled from 3 independent experiments $(n=20 \sim 30)$. Error bars represent the mean \pm SEM. ( $\mathbf{D}$ and $\mathbf{E})$ ${ }^{*} P<0.05$, by 2 -way ANOVA and 2-tailed Student's $t$ test.

The TIA1-N357S variant promotes LLPS and impairs SG clearance. To examine the impact of the TIA1-N357S (NS) mutation on LLPS, we constructed a phase diagram by measuring the coexistence line of a protein-depleted light phase and a protein-enriched dense phase as a function of temperature and protein concentration. Like the previously characterized TIA1-E384K (EK) mutant, the TIA1-NS mutation caused a significant leftward shift in the coexistence line to a lower protein concentration, indicating an increased propensity of mutant TIA1 to phase separate as a result of stronger intermolecular protein-protein interactions (Figure 2A). Although the promotion of LLPS with TIA1-EK was associated with an increased rate of amyloid-like fibril formation, as demonstrated by a time-dependent increase in thioflavin- $T$ incorporation when compared with TIA1WT, we did not see this increase with purified TIA1-NS (Figure 2B). 
A

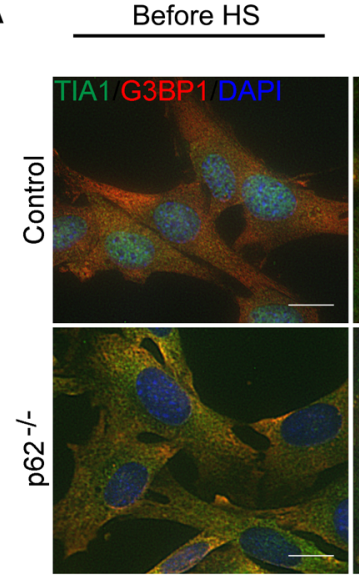

C

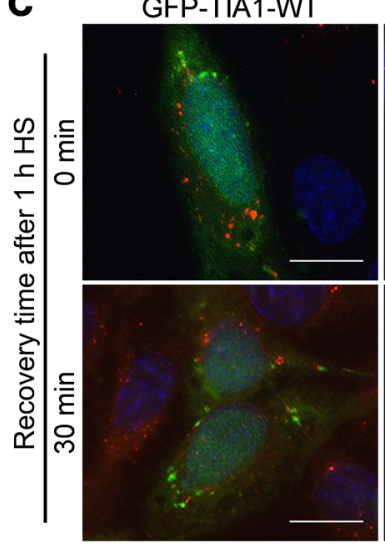

Recovery time after $1 \mathrm{~h} \mathrm{HS}$
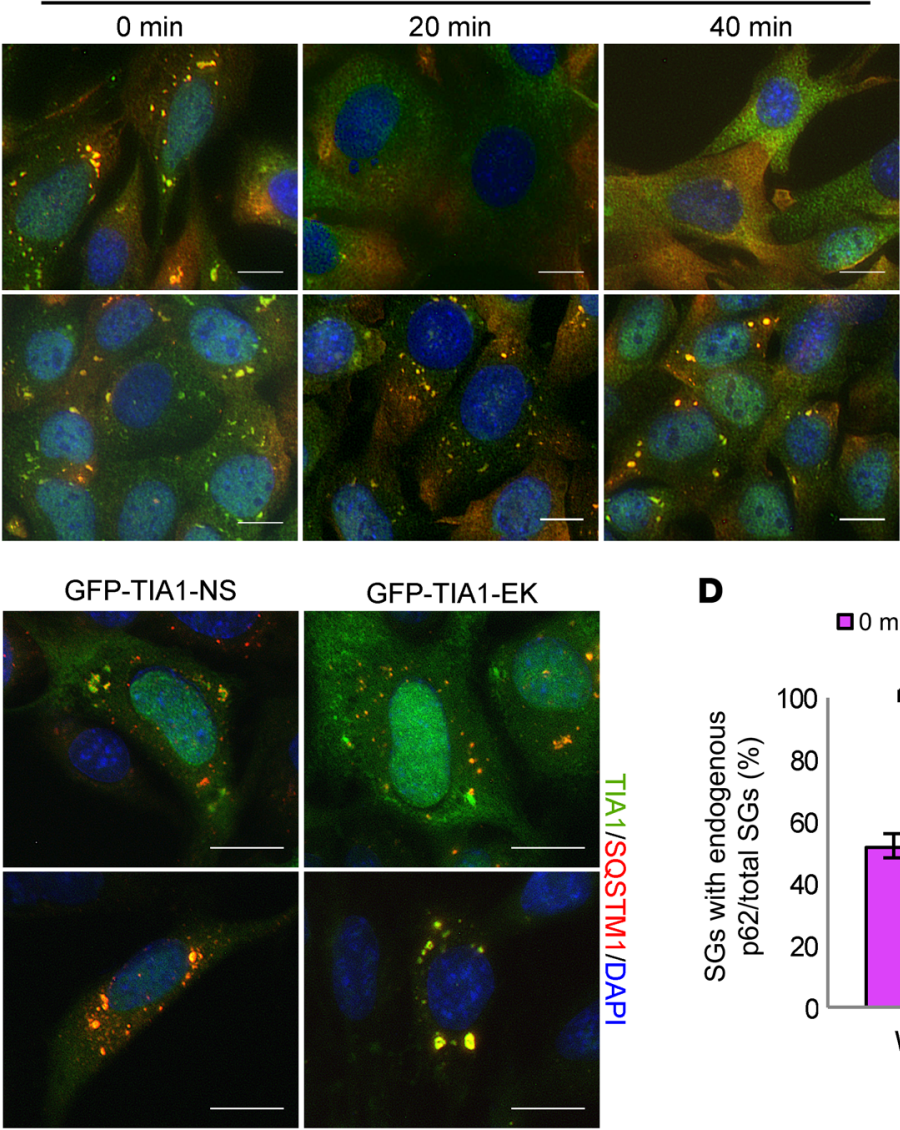

D

B
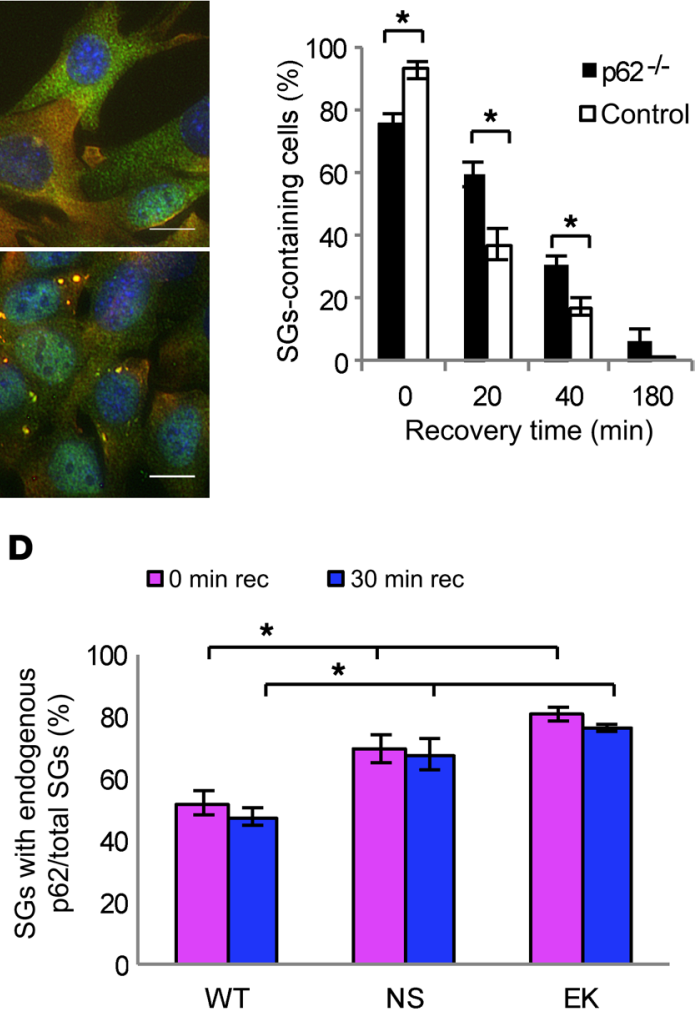

Figure 3. SQSTM1 is necessary for SG homeostasis. (A) Immunofluorescence images of control or SQSTM1-knockout MEFs ( $p 62^{-/-}$) incubated at $42^{\circ} \mathrm{C}$ for 1 hour and returned to $37^{\circ} \mathrm{C}$ for the indicated durations followed by immunostaining for TIA1 (green) and G3BP1 (red) to detect SGs. (B) Graph of the percentage of cells containing TIA1/G3BP1-positive SGs as in A. Transfected cells were counted and are indicated as the total number of cells. Representative data were pooled from 3 independent experiments ( $n=150 \sim 200)$. (C) Immunofluorescence images of MEFs expressing GFP-TIA1-WT, -NS, or -EK and immunostained with SQSTM1 antibody following incubation at $42^{\circ} \mathrm{C}$ for 1 hour and reincubation at $37^{\circ} \mathrm{C}$ for the indicated durations. (D) Bar graph of the percentage of GFP-TIA1/SQSTM1-positive SGs in C. Individual GFP-TIA1 SCs were counted and are indicated as the total number of SGs. Representative data were pooled from 3 independent experiments $(n=800 \sim 1000)$. Scale bars: $5 \mu$ m. Error bars represent the mean \pm SEM. (B and $\mathbf{D}){ }^{*} P<0.05$ by 2-way ANOVA and 2-tailed Student's $t$ test.

The effect of TIA1-NS and -EK variants on LLPS in vitro suggested that these variants might affect the dynamics of SGs in vivo. To test this hypothesis, we expressed GFP-TIA1-WT, -NS, or -EK in mouse embryonic fibroblasts (MEFs) for 24 hours. Immunoblotting confirmed similar levels of expression for GFP-TIA1WT, -NS, and -EK (Supplemental Figure 2A). MEFs expressing GFP-TIA1 were fixed and stained with the SG marker G3BP1. Untreated GFP-TIA1-WT-expressing cells did not form SGs, while some SGs were detected in GFP-TIA1-NS- or -EK-expressing cells (Figure 2, C and D). Heat shock (HS) at $42^{\circ} \mathrm{C}$ induced SG formation in MEFs expressing GFP-TIA1-WT, -NS, or -EK after 1 hour. Upon return to $37^{\circ} \mathrm{C}$ for 30 minutes, approximately $10 \%$ of GFP-TIA1-WT-expressing MEFs contained SGs as compared with approximately $40 \%$ of MEFs expressing GFP-TIA1-NS or -EK (Figure 2, C and D). TIA1 in SGs is normally in rapid equilibrium with TIA1 in the surrounding cytoplasm. We investigated whether TIA1-NS mutation affects its mobility within SGs. MEFs expressing GFP-TIA1-WT, -NS, or -EK were treated with $0.5 \mathrm{mM}$ arsenite (AsIII) for 1 hour, and GFP-TIA1 SGs were subjected to fluorescence recovery after photobleaching (FRAP). Following photobleaching, approximately $65 \%$ of GFP-TIA1-WT fluorescence rapidly recovered, whereas GFP-TIA1-NS and -EK showed approximately $40 \%$ and $60 \%$ recovery, respectively (Figure $2 \mathrm{E}$ and Supplemental Figure 2B).

SQSTM1 participates in SG protein clearance. To explore the role of SQSTM1 in SG formation and clearance, we immunostained endogenous TIA1 and G3BP1 in control MEFs and MEFs lacking SQSTM1 ( $p 62^{-/-}$MEFs). Untreated control or $p 62^{-/-}$MEFs did not form TIA1/G3BP1-positive SGs (Figure 3, A and B). Following 1 hour of HS, approximately $95 \%$ of control MEFs and $70 \%$ of $p 62^{-/-}$ MEFs contained SGs (Figure 3, A and B). Twenty minutes after reincubating the cells at $37^{\circ} \mathrm{C}$, approximately $50 \%$ of the SGs disappeared in the control MEFs and ultimately dissipated by 1 hour. In contrast, $p 62^{-/-}$MEFs had a slower SG clearance, with some cells maintaining SGs after 180 minutes (Figure 3, A and B). Persistent TIA1-positive SGs in $162^{-/-}$MEFs coimmunolocalized with an antibody that recognizes ubiquitin conjugates (Supplemental Figure 3A). Consistent with SQSTM1 mediation of the clearance of SGs 
A

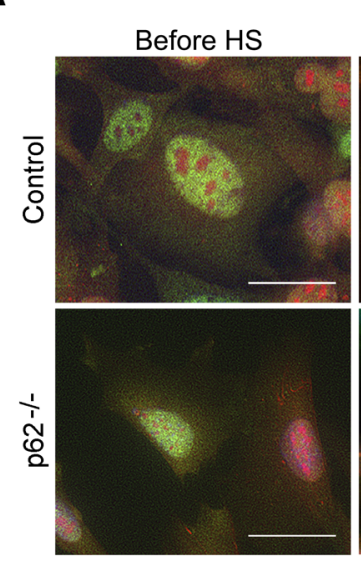

C

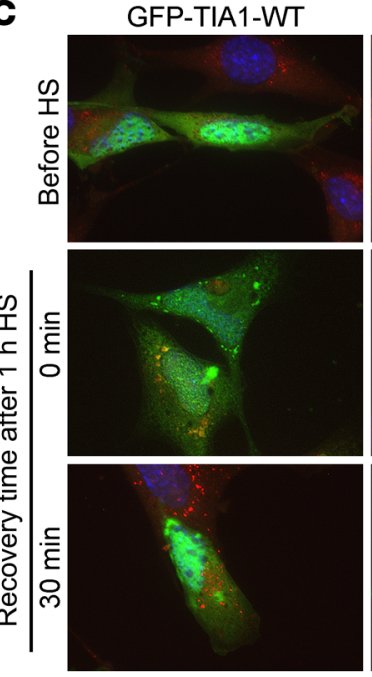

Recovery time after $1 \mathrm{~h} \mathrm{HS}$

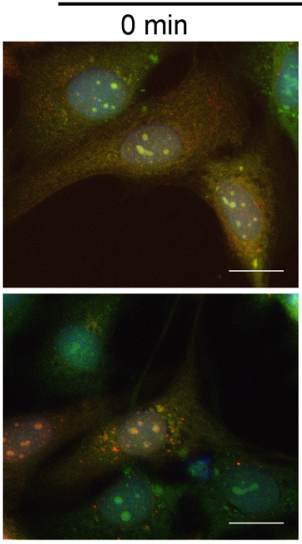

GFP-TIA1-NS

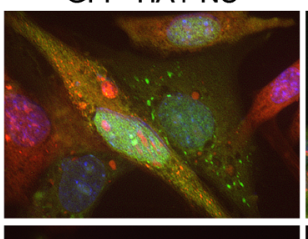

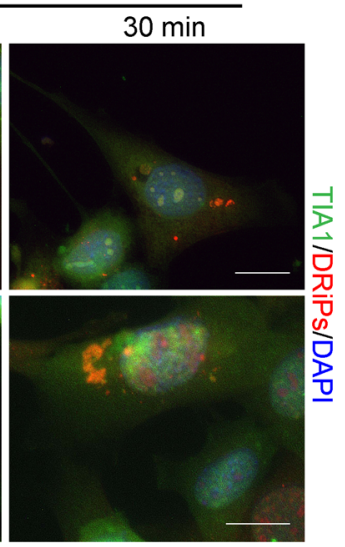

GFP-TIA1-EK

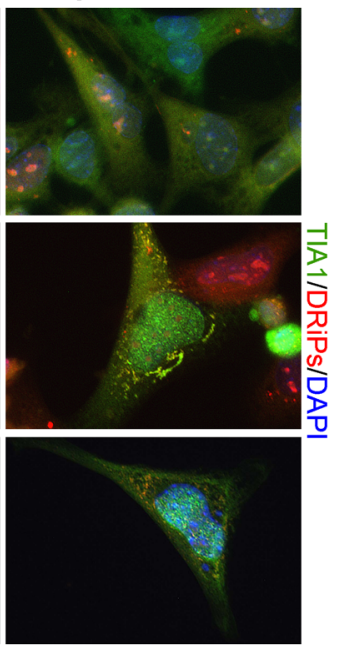

B

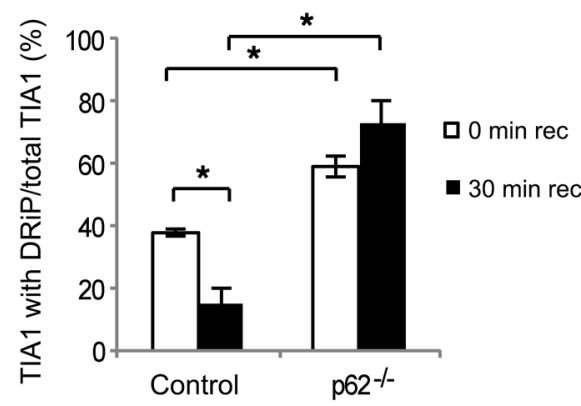

D

口Before HS $\quad$ a $0 \mathrm{~min}$ rec $\quad \square 30 \mathrm{~min}$ rec

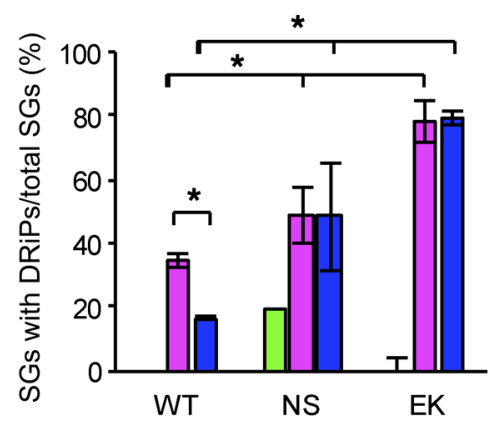

Figure 4. The presence of aggregated proteins increases TIA SG persistence in the presence of TIA1 mutations or loss of SQSTM1. (A) Immunofluorescence images of control or $p 62^{-/-}$MEFs labeled with Alexa Fluor 594-azide (red) to detect DRiPs after incubation at $42^{\circ} \mathrm{C}$ for 1 hour and following a 30-minute HS recovery. Representative data were pooled from 3 independent experiments $(n=350 \sim 450)$. Scale bars: $5 \mu \mathrm{m}$. (B) Graph of the percentage of TIA1 SGs labeled with Alexa Fluor 594-azide (red) detecting DRiPs in control or SQSTM1-knockout MEFs ( $p 62^{--}$), incubated at $42^{\circ} \mathrm{C}$ for 1 hour and returned to $37^{\circ} \mathrm{C}$ for 30 minutes. Individual TIA1 SGs (green) were counted and are indicated as the total number of TIA1 SGs. Representative data were pooled from 3 independent experiments $(n=350 \sim 450)$. (C) IF images of MEFs expressing GFP-TIA1-WT, -NS, or -EK and labeled with Alexa Fluor 594-azide (red) to detect DRiPs before $\mathrm{HS}$, after incubation at $42^{\circ} \mathrm{C}$ for 1 hour, and following a 30 -minute HS recovery period. Representative data were pooled from 3 independent experiments $(n=350 \sim 450)$. (D) Bar graph of the percentage of TIA1 SGs labeled with DRiPs as in C. Individual TIA1 SGs (green) were counted and are indicated as the total number of TIA1 SGs. ${ }^{*} P<0.05$ by 2-way ANOVA and 2-tailed Student's $t$ test.

via an autophagy-lysosomal pathway, we found that incubation of $p 62^{-1}$ MEFs with the lysosomal inhibitor bafilomycin A during HS recovery did not further augment the decrease in SG clearance seen with loss of SQSTM1 (Supplemental Figure 3B).

To determine whether there is a direct connection between TIA1 and SQSTM1, we immunostained for endogenous SQSTM1 in MEFs expressing GFP-TIA1-WT, -NS, or -EK after 1 hour of HS and following a 30 -minute recovery to $37^{\circ} \mathrm{C}$. We found that a subset of GFP-TIA1-WT SGs colocalized with SQSTM1. Colocalization of SQSTM1 and GFP-TIA1 was greater in GFP-TIA1-NS- and -EK-expressing MEFs than in GFP-TIA1-WT-expressing MEFs (Figure 3, C and D).

Defective ribosomal products (DRiPs) are prematurely terminated and/or misfolded polypeptides $(28,32)$. They may form transient inclusions that colocalize with SQSTM1 and are target- ed for clearance via the proteasome or autophagy (28, 32-34). DRiPs may also accumulate adjacent to and within SGs, which may lead to altered SG kinetics $(28,32)$. To determine whether SQSTM1 mediates the clearance of DRiP-containing SGs, we treated control and $p 62^{--}$MEFs with OP-puromycin to generate and label DRiPs. After 1 hour of HS, approximately 30\% of SGs colocalized with DRiPs in control MEFs, and approximately $45 \%$ of SGs colocalized with DRiPs in $p 62^{--}$MEFs (Figure 4, A and B). Surprisingly, after 30 minutes of HS recovery, DRiP-containing SGs significantly decreased in control MEFs, but DRiP-containing SGs accumulated in $p 62^{-/}$MEFs, suggesting that the clearance of aberrant SGs with DRiPs is SQSTM1 dependent (Figure 4, A and B). Likewise, we treated MEFs expressing GFP-TIA1-WT, -NS, or -EK with OP-puromycin to generate and label DRiPs as above. Following 1 hour of HS, approximately $35 \%$ of the GFP-TIA1-WT 
A

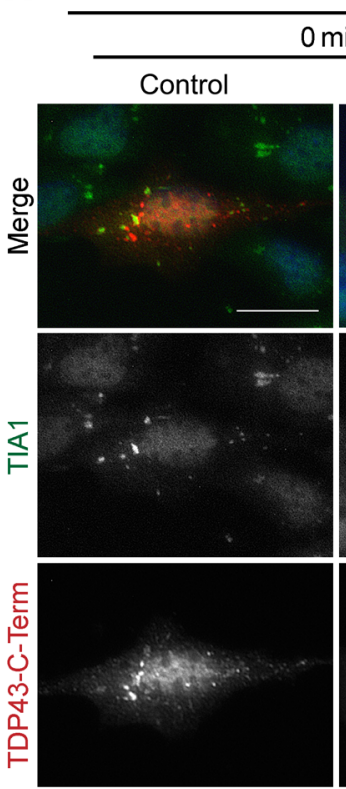

Recovery time after $1 \mathrm{~h} \mathrm{HS}$

$\mathrm{p} 62^{-/-}$
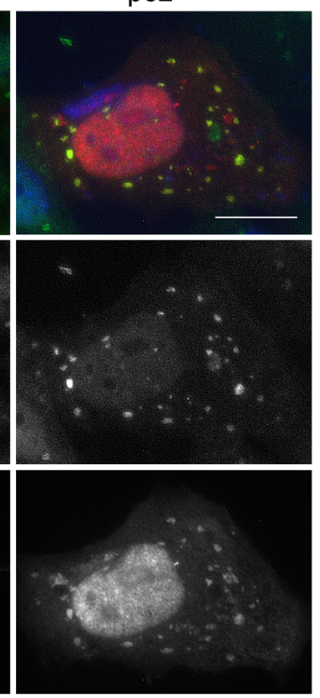

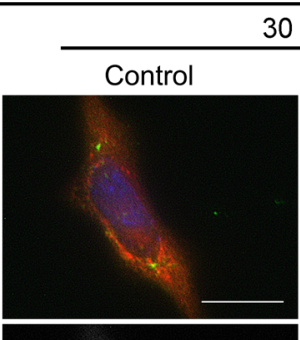

$30 \min$

$\mathrm{p} 62^{-/-}$
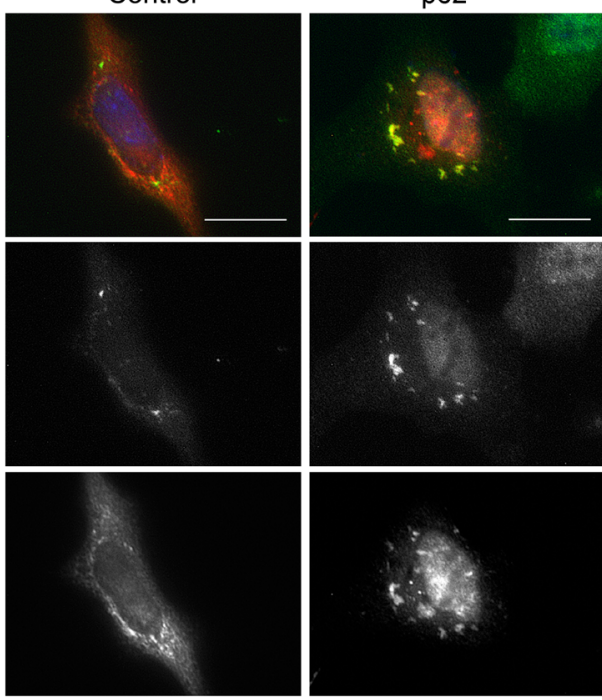

B

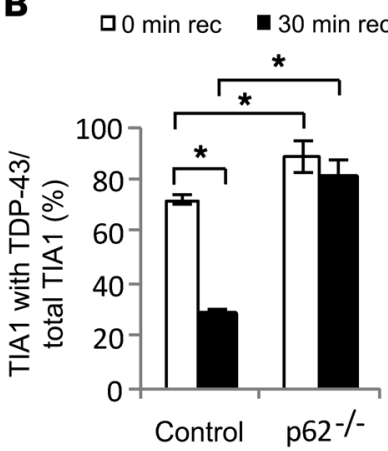

Figure 5. TDP-43 aggregates increase TIA SG persistence in the loss of SQSTM1. (A) Immunofluorescence images of endogenous TIA1 (green) from control or $p 62^{-1-}$ MEFs transfected with an mCherry-tagged TDP-43 C-terminal fragment (red) after incubation at $42^{\circ} \mathrm{C}$ for 1 hour and following a 30 -minute $\mathrm{HS}$ recovery. Representative data were pooled from 3 independent experiments $(n=350 \sim 450)$. (B) Bar graph showing the percentage of TIA1 SGs with mCherry TDP-43 C-terminal fragment in control or p62-/- MEFs from A. Individual TIA1 SGs (green) were counted and are indicated as the total number of TIA1 SGs. DAPI nuclear staining is shown in blue. Scale bars: $5 \mu \mathrm{m}$. Error bars represent the mean \pm SEM. ${ }^{*} P<0.05$, by 2-way ANOVA and 2-tailed Student's $t$ test.

SGs colocalized with DRiPs, and this number decreased upon HS recovery (Figure 4, C and D). In contrast, GFP-TIA1-NS and -EK SGs colocalized more robustly with DRiPs following HS, and this association persisted upon HS recovery (Figure 4, C and D).

We reasoned that since DRiPs were misfolded proteins, then TIA1 may similarly accumulate with other misfolded and aggregated proteins at SGs. To test this, we expressed an aggregation-prone $25-\mathrm{kDa}$ C-terminal TDP-43 fragment with an mCherry tag (mCherry-TDP-CTF) in control and $p 62^{-/-}$MEFs and subjected them to 1 hour of HS followed by 30 minutes of recovery. Immunofluorescence (IF) for endogenous TIA1 revealed that, upon HS, TIA1 and mCherry-TDP-CTF colocalized in both cell types (Figure 5 , A and B). However, upon recovery in the $p 62^{-/-} \mathrm{MEFs}$, approximately $80 \%$ of the persistent TIA1-positive SGs also contained mCherry-TDP-CTF as compared with approximately $20 \%$ of the SGs in control MEFs (Figure 5, A and B).

MSP-associated SQSTM1 mutations impair TIA1-positive SG homeostasis. To determine whether SQSTM1 disease mutations identified in MSP patients affected the clearance of TIA1-containing SGs, MEFs expressing mCherry, mCherry-SQSTM1-WT, or mCherry-SQSTM1 carrying the MSP mutation SQSTM1-P392L (PL), SQSTM1-M404V (MV), or SQSTM1-A390X (AX) were immunostained with antibodies against endogenous TIA1. Without HS, MEFs expressing mCherry or mCherry-SQSTM1-WT did not form SGs (Figure 6A). In contrast, even without HS, MEFs expressing the mCherry-SQSTM1 disease mutations (PL, MV, or AX) had TIA1-positive SGs (Figure 6A). As expected, most cells contained SGs following 1 hour of HS, and after 30 minutes of post-HS recovery, approximately $50 \%$ of SGs were cleared from MEFs expressing mCherry or mCherry-SQSTM1-WT. In contrast, following a 30-minute HS recovery, MEFs expressing mCherry-SQSTM1-PL, -MV, or -AX had persistent SGs in $80 \%$ of cells, suggesting that disease-associated SQSTM1 mutations delay SG clearance (Figure 6A).

To explore the synergy of a SQSTM1 disease mutation and the TIA1-N357S variant, we used patient-derived fibroblasts from 2 control patients (fibroblast lines 112 and 409), 2 patients carrying the TIA1-N357S variant (fibroblast lines 107 and 319), and 1 patient carrying both SQSTM1 c.1165+1 G>A mutations, which generate a truncated SQSTM1-A390X mutation and a TIA1-N357S variant (fibroblast line 483). We treated low-passage fibroblasts with 0.5 $\mathrm{mM}$ AsIII for 1 hour and then replaced AsIII-containing media for 40 minutes. Fibroblasts were immunostained for endogenous TIA1 and G3BP1. Untreated fibroblasts had no detectable SGs. Following 1 hour of AsIII treatment, all fibroblasts formed TIA1/ G3BP1-positive SGs (Figure 6, B and C). These SGs dissipated in all fibroblasts but were significantly increased in fibroblast line 483, which contained both disease variants (Figure 6, B and C).

Coexpression of MSP-associated SQSTM1 mutations and TIA1N357S is myotoxic. To see whether the synergistic effect of SQSTM1 mutations and TIA1 variants occurred in muscle cells, we cotransfected C2C12 myoblasts with plasmids expressing mCherrySQSTM1-WT or mCherry-SQSTM1 carrying an MSP mutation (PL, MV, or AX) and GFP-TIA1-WT or GFP-TIA1 carrying a distal myopathy variant (EK or NS). After 60 minutes of HS, approximately $100 \%$ of myoblasts had TIA1-GFP-positive SGs, regardless of the mutation or variant (Figure 6D). In contrast, after 60 minutes of HS recovery, myoblasts expressing a disease-associated SQSTM1 mutation with a TIA1 variant had an increase in persistent SGs as compared with that seen in mCherry-SQSTM1-WT-expressing myoblasts (Figure 6D). To determine whether coexpression of 
A

a Before HS $\quad 0 \mathrm{~min} \mathrm{rec} \quad \square 30 \mathrm{~min} \mathrm{rec}$

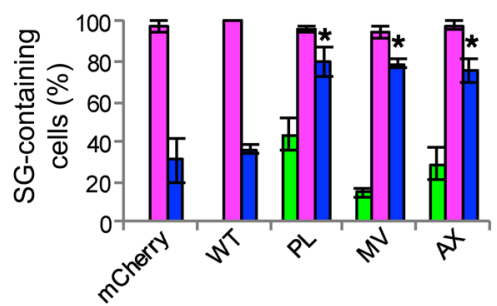

B

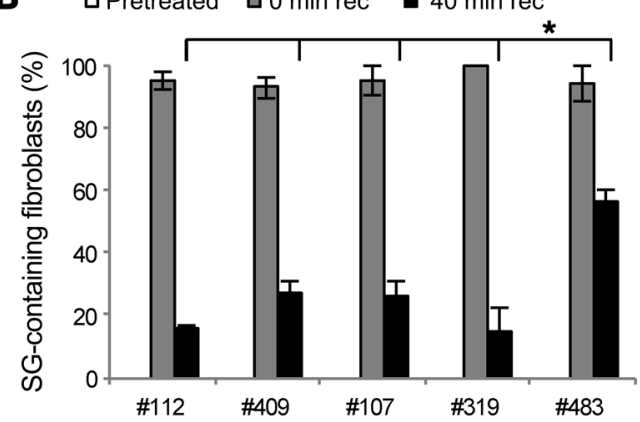

C
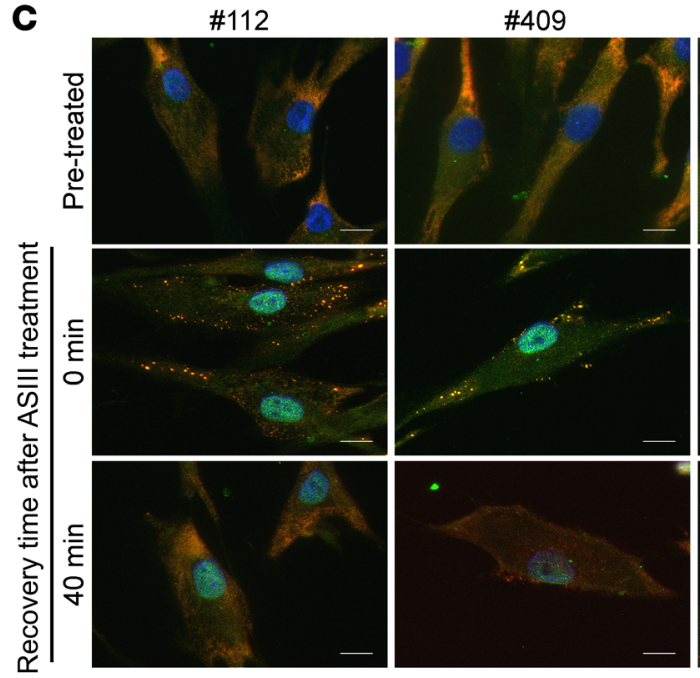

$\square 0 \mathrm{~h} \mathrm{rec} \square 1 \mathrm{~h} \mathrm{rec}$

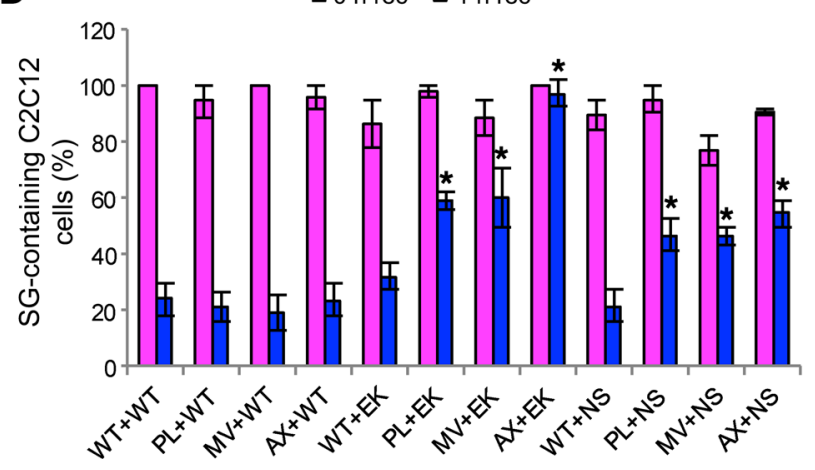

\#107

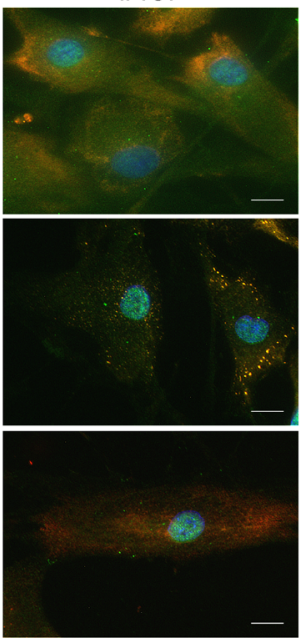

\#319

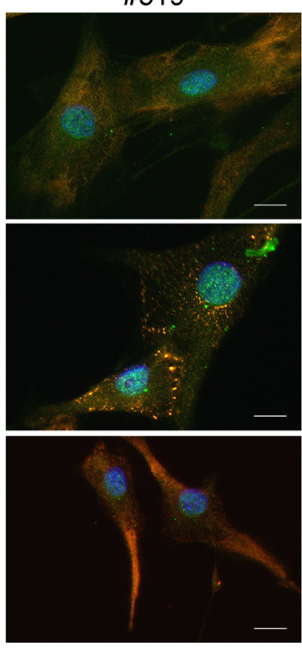

\#483

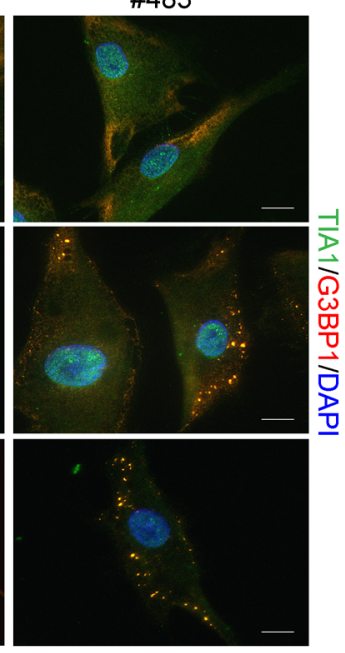

E

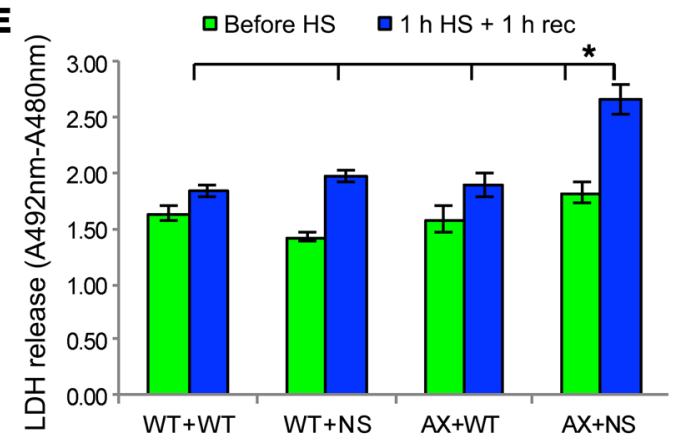

Figure 6. SQSTM1 disease mutations alter SG kinetics and synergistically mediate myotoxicity with TIA1-N357S. (A) Bar graph showing the percentage of MEFs containing endogenous TIA1-positive SGs. MEFs were transfected with mCherry, mCherry-SQSTM1-WT, or 1 of 3 different disease mutations ( $\mathrm{PL}, \mathrm{MV}$, or $\mathrm{AX}$ ) incubated at $42^{\circ} \mathrm{C}$ for 1 hour and subsequently returned to $37^{\circ} \mathrm{C}$ for the indicated durations. Transfected cells were counted and are indicated as the total number of cells. Representative data were pooled from 3 independent experiments $(n=450 \sim 550)$. (B) Bar graph showing the percentage of fibroblasts containing TIA1/C3BP1-positive SGs from fibroblasts of control patients (fibroblast lines 112 and 409 ), patients carrying the TIA1-N357S variant (fibroblast lines 107 and 319), and a patient carrying both a SOSTM1-A390X mutation and a TIA1-N357S variant (fibroblast line 483) immediately following $0.5 \mathrm{mM}$ AsIII treatment for 1 hour or following a 40 -minute recovery. (C) Immunofluorescence images of patients' fibroblasts detailed in B, immunostained with TIA1 (green) and G3BP1 antibodies (red) before, immediately following 0.5 mM AsIll treatment for 1 hour, and following a 40-minute recovery. DAPI nuclear staining is shown in blue. Scale bars: $5 \mu \mathrm{m}$. Representative data were pooled from 3 independent experiments $(n=120 \sim 150)$. (D) Bar graph showing the percentage of C2C12 myoblasts containing TIA1-positive SCs. C2C12 myoblasts were cotransfected with mCherry, mCherry-SQSTM1-WT, or mCherry-SQSTM1 with 1 of 3 different disease mutations (PL, MV, or AX) and GFP-TIA1-WT or 1 of 2 variants (EK or NS) incubated at $42^{\circ} \mathrm{C}$ for 1 hour and subsequently returned to $37^{\circ} \mathrm{C}$ for the indicated durations. (E) Bar graph of $\mathrm{LDH}$ release from C2C12 myoblasts similar to those in $\mathbf{D}$, before HS and after 1 hour of HS, with an additional 1-hour recovery at $37^{\circ} \mathrm{C}$. The absorbance of the samples was measured at $492 \mathrm{~nm}$. The reference wavelength at $680 \mathrm{~nm}$ was measured. Representative data were pooled from 3 independent experiments $(n=150 \sim 200)$. Error bars represent the mean \pm SEM. ${ }^{*} P<0.05$, by 2-way ANOVA and 2-tailed Student's $t$ test. 
SQSTM1-AX and TIA1-NS synergistically enhanced myotoxicity, we performed a lactate dehydrogenase (LDH) release assay on C2C12 myoblasts cotransfected with plasmids expressing mCherrySQSTM1-WT or mCherry-SQSTM1-AX and GFP-TIA1-WT or GFPTIA1-NS before HS and after 60 minutes of HS with an additional 60 minutes of HS recovery, a time point at which persistent SGs are present. Consistent with a synergistic effect, SQSTM1-AX- and TIA1-NS-expressing myoblasts had increased LDH release as compared with the SQSTM1-WT or TIA1-WT transfectants (Figure 6E).

\section{Discussion}

The present study identified 14 patients from 9 families with distal myopathy and RV-IBM pathology, who all carried a previously reported pleotropic mutation in SQSTM1 and a rare TIA1-N357S variant. These patients did not manifest other phenotypes associated with SQSTM1 mutations such as PDB, ALS, or FTD at the time of examination. Importantly, SQSTM1 and TIA1 accumulated with TDP-43 in patients' muscle tissue, suggesting that they participate in the disease pathogenesis. While the connection between SG clearance and autophagic protein degradation has been previously suggested, the present study provides human genetic evidence for a pathologic interaction $(27,28)$. These data support the notion that inheritance of a TIA1 variant can shift the phenotypic spectrum of SQSTM1 mutations to myodegeneration.

Mutations in SQSTM1 were initially identified in patients with familial PDB and included the SQSTM1-P392L and c.1165+1 G>A splice acceptor mutations (12). Notably, the SQSTM1-P392L mutation was found in approximately $9 \%$ of patients with sporadic $\mathrm{PDB}$, making it the most common genetic cause of the disease (12). Later reports identified other missense mutations in or truncations of the UBA domain of SQSTM1, including the M404V mutation as causative in familial and sporadic PDB (29). Subsequently, SQSTM1 mutations including the P392L and c.1165+1 G>A were identified in patients with familial ALS and/or FTD (13, 14, 35). In 2015, we described 3 patients who had distal myopathy and RV-IBM pathology with the SQSTM1 c.1165+1 G>A mutation (1). Since the same SQSTM1 mutation can generate distinct phenotypes in different patients, we suggested that SQSTM1-associated disease be termed multisystem proteinopathy type 4 (MSP4) (1). MSP defines a pleotropic genetic disease, in which a single gene and mutation can manifest as distinct disease phenotypes that include PDB, RV-IBM, ALS, and FTD.

We previously reported a TIA1-E384K founder mutation as the cause of WDM, an autosomal dominant distal myopathy with RV-IBM pathology (6). The phenotype of patients with WDM is similar to that of the SQSTM1-TIA1 patients described in this report. WDM is a progressive, late-onset myopathy that typically manifests in the fifth decade. A very small number of WDM patients who are homozygous for the TIA1-E384K variant have an earlier onset and more rapid disease progression, suggesting that the TIA1-E384K mutation exerts a dose-dependent, dominant effect (36). Similarly, we identified 1 patient in our cohort with distal myopathy who was homozygous for the TIA1-N357S variant. This patient did not have a second mutation in SQSTM1. The TIA1-N357S variant was overrepresented in our myopathy cohort and was present in $20 \%$ of our distal myopathy patients. These data support the idea that the TIA1-N357S variant may itself be a risk factor for distal myopathy.
The findings in our study may relate to other forms of MSP. Specifically, mutations in VCP, a ubiquitin segregase necessary for autophagic protein degradation, were the first identified cause of MSP (originally termed IBMPFD, for inclusion body myopathyassociated with Paget's disease of the bone and fronto-temporal dementia) (2). Recent studies confirm the original report that $90 \%$ of patients develop RV-IBM, 42\% develop PDB, and 30\% develop FTD, but also expand the degenerative phenotypes to include $9 \%$ of patients with ALS and $4 \%$ with parkinsonism (37). Why some patients with VCP mutations develop RV-IBM and others PDB is not clear, but as with our study, it probably relates to oligogenic inheritance. For example, patients manifesting with VCP-associated dementia are more likely to carry an APOE4 allele (38). Some VCP mutations, like the SQSTM1-P392L mutation have low MAFs in the general population. Rare variants in VCP and SQSTM1 are identified as overrepresented in large cohorts of patients with sporadic $\mathrm{PDB}$, IBM, ALS, or dementia, suggesting that they may be risk factors for disease or have reduced penetrance (13, 14, 30, 39-43).

Digenic inheritance can manifest in several ways. For example, the inheritance of a second genetic variant can affect the primary gene's mutant phenotype by modifying its severity or age of onset. Another example occurs when a single genetic mutation may have a reduced penetrance, and the inheritance of a mutation in a second gene unmasks the variable expressivity of the primary mutation. Examples of true digenic inheritance, in which the inheritance of 2 variants on 2 different genes is necessary to manifest disease, are rare and challenging to prove. One recent example is found in some forms of facioscapulohumeral muscular dystrophy type 2 (FSHD2), in which a patient must inherit both a permissive D4Z4 allele and an SMCHD1 variant on different chromosomes (44). The strength of this example relates to its presence in multiple families and the convincing mechanistic interaction between the 2 alleles.

While autophagy has been implicated in SG clearance, this is the first report to our knowledge to demonstrate a role for SQSTM1 in SG dynamics. RNA-binding proteins such as TIA1 initiate granule formation via their LCDs by promoting reversible LLPS (45). Persistent SGs may occur when LLPS converts the LCD into an irreversible amyloid-like structure (20). Our data suggest that, while the TIA-EK and -NS variants promote phase separation, only the TIA1-EK variant enhances its amyloidogenic conversion. This may explain why heterozygous TIA1-E384K mutations lead to WDM, yet TIA1-N357S variants require a SQSTM1-mutant environment for penetrance. Loss of SQSTM1 or SQSTM1-mutant expression leads to the accumulation of undegraded, ubiquitinated, and insoluble proteins (46). The presence of misfolded and aggregate-prone proteins increases SG formation and persistence in cell culture (32, 47). Likewise, we found that in the setting of SQSTM1-knockout or -mutant expression, TIA1-persistent SGs colocalized with aggregated proteins such as DRiPs and a C-terminal TDP-43 fragment. While it is possible that SQSTM1 directly shuttles ubiquitinated and aggregated TIA1 to the autophagosome, we propose an alternate model. Specifically, the presence of the SQSTM1 mutations leads to the cytosolic accumulation of aggregated proteins that then serve as a nidus for RNA granule conversion to an irreversible state.

The mechanism whereby disturbances in RNA granule dynamics and persistence of poorly dynamic SGs impair cell function in general or cause muscle degeneration in particular remains 
unclear. One possibility is that disturbance of the SG dynamics results in the impairment of functions normally conducted within these complex, membrane-less organelles (e.g., remodeling of messenger ribonucleoprotein particles [mRNPs] or participating in intracellular signaling cascades). Alternatively, the condensed liquid environment of the SG may promote untoward fibrillization of RNA-binding proteins to produce insoluble, pathological species. Indeed, in vitro LLPS promotes a rapid fibrillization of RNA-binding proteins that occurs within the condensed liquid phase, resulting in the accumulation of potentially toxic species (20).

An enigmatic feature of the syndrome MSP and most of the constituent diseases (ALS, FTD, and RV-IBM) is the prominence of TDP-43 pathology, despite tremendous heterogeneity in its genetic etiology. Thus, mutations in genes as diverse as VCP, SQSTM1, C9ORF72, HNRNPA1, HNRNPA2B1, TARDBP, and TIA1 culminate in highly similar histopathology, in which cytoplasmic deposition of fibrillar TDP-43 is a prominent feature. Notably, even when disease mutations occur in other RNA-binding proteins prone to fibrillization, such as TIA1, the histopathological picture is dominated by TDP-43 pathology (7). A likely explanation for this phenomenon is that there are differences in the stability of fibrils assembled from various RNA-binding proteins. Indeed, it was recently demonstrated that recruitment of TDP-43 to SGs results in an abrupt reduction in the mobility of this protein and conversion to an SDS-resistant, poorly soluble species, whereas other RNA-binding proteins recruited to the same SGs remain mobile and soluble (7).

Cytosolic TDP-43 accumulation has been shown to elicit cellular toxicity via several mechanisms including disruptions in RNA transport, splicing, and nucleocytoplasmic shuttling (48). Interestingly, autophagic inhibition leads to the cytosolic accumulation in TDP-43 (49). This effect also occurs in differentiated skeletal muscle, further connecting autophagic impairment with SG pathology(49). Interestingly, enhancing autophagy has been shown to be protective in the setting of TDP-43 accumulation (50).

MSP may represent an alternative manifestation of digenic inheritance. Specifically, a mutation on gene X (i.e., SQSTM1 or $V C P$ ) can lead to variable expressivity of multiple phenotypes (PDB, ALS, FTD, or RV-IBM). Inheritance of a variant on gene Y (TIA1), while impenetrant alone, dictates the phenotypic expression of gene $\mathrm{X}$ (distal myopathy with RV-IBM pathology). This model explains the genetics of MSP but may also relate to the phenotypic penetrance seen within the ALS-FTD spectrum. In addition, our model supports a pathomechanistic connection between SG homeostasis and ubiquitin-mediated autophagic degradation.

\section{Methods}

Genetic studies. A cohort of 1,293 patients with presumed hereditary myopathy were sequenced using a targeted high-throughput sequencing panel called MYOcap. The panel covered the exons, as well as some 3 '- and $5^{\prime}$-UTRs of selected genes either reported to cause muscle disease or to be functionally related to such genes (15). The MYOcap panel has been regularly updated, and samples of the cohort were sequenced with 4 versions covering 180, 236, 265, and 297 genes, respectively. The TIA1 and SQSTM1 genes were included in all versions. Specific probes were manufactured by Roche NimbleGen (SeqCap EZ Choice Library). DNA was extracted from blood by standard methods, and enrichment and sequencing were performed at the Institute for Molecular Medicine of Finland (FIMM), Biomedicum in Helsinki, Finland, and at the Wellcome Trust Centre for Human Genetics (WTCHG) in Oxford, United Kingdom, using the Illumina HiSeq1500 and HiSeq2000 platforms. The sequencing raw data were mapped against the human reference genome GRCh37/hg19. Data analysis was performed using an in-house pipeline as described earlier (15).

Indexed genomic DNA (gDNA) libraries were prepared from gDNA using a TruSeq DNA Preparation Kit (Illumina), and exome capture was done using a TruSeq Exome Enrichment Kit (Illumina) according to the manufacturer's protocol. Sequencing was performed with 100bp paired-end reads on a HiSeq2000 (Illumina). Reads were aligned to the human reference genome with NovoAlign or Burrows-Wheeler Aligner. Variants were called with SAMtools (http://samtools.sourceforge.net/) and annotated with SeattleSeq (http://snp.gs.washington. edu/SeattleSeqAnnotation138/). Coverage across genomic intervals was calculated using bedtools (http://bedtools.readthedocs.io/en/ latest/). Genomic coordinates for regions targeted by the whole-exome capture kit were provided by Illumina. High-throughput sequencing results were verified by Sanger sequencing. Allele frequencies for the general population were taken from gnomAD (51).

Protein purification, in vitro fibrillization, and phase diagram. Recombinant DNA for TIA1-WT, N387S, and E384K constructs were cloned into the pETite N-His SUMO Kan vector (Lucigen). Plasmids were chemically transformed into BL21_DE3 cells (Lucigen). Protein purification, in vitro fibrillization, and phase diagram protocols described in Mackenzie et al. (7) were followed. For the phase diagram, the spectrum of BSA (nonamyloid fibril-forming) was measured as a baseline. That baseline was subtracted from all the WT, E384K, and N357S spectra at each time point, respectively.

Muscle pathology, reagents, and antibodies. Frozen muscle sections were processed for routine histochemical analysis, including H\&E, modified Gomori trichrome, combined succinate dehydrogenasecytochrome oxidase, ATPase at $\mathrm{pH}$ 9.2, $\mathrm{pH} 4.3$ and reduced nicotinamide adenine dinucleotide-tetrazolium reductase.

The following antibodies were used: anti-TIA1 polyclonal antibody (C-20; Santa Cruz Biotechnology; catalog sc-1751; 1:500 for WB, 1:100 for IF); anti-G3BP1 polyclonal antibody (Proteintech; catalog 13057-2-AP; 1:100 for IF); anti-p62 polyclonal antibody (Proteintech; catalog 18420-1-AP; 1:1,000 for WB and 1:100 for IF); anti-GFP polyclonal antibody (MilliporeSigma; catalog G1544; 1:500 for WB); anti-Ub monoclonal antibody (FK2, Biomol; catalog PW-8810; 1:500 for WB and 1:100 for IF); anti-Ub polyclonal antibody (Dako; catalog Z0458; 1:5,000 for WB and 1:100 for IF); and anti-GAPDH polyclonal antibody (Cell Signaling Technology; catalog 2118; 1:1,000 for WB). For the detection of endogenous proteins in patients' muscle biopsies, the following antibodies were used for IF: anti-TIA1 polyclonal antibody (Abcam; catalog ab61700; 1:100) and anti-p62 polyclonal antibody (MilliporeSigma; catalog P0067; 1:100).

Cell culture and transient transfection. Fibroblasts were cultured in Fibroblast Basal Medium FGM-2 (Lonza; catalog CC-313) supplemented with FGM-2 SingleQuot Kit \& Growth Factors (Lonza; catalog CC-4126), as recommended by the manufacturer, at $37^{\circ} \mathrm{C}$ and $5 \% \mathrm{CO} 2$ in a humidified incubator. Immortalized control and $p 62^{---}$MEFs were provided by M. Komatsu (Niigata University, Niigata, Japan). MEFs were maintained in DMEM (Gibco, Thermo Fisher Scientific; cata$\log$ 11965-084); 10\% FBS (Atlanta Biologicals; S10350H); $50 \mu \mathrm{g} / \mathrm{ml}$ 
penicillin-streptomycin (MilliporeSigma; catalog P4333); 1\% sodium pyruvate (Gibco, Thermo Fisher Scientific; catalog 11360070); and 1\% nonessential amino acids (Gibco, Thermo Fisher Scientific; catalog $11140050)$ at $37^{\circ} \mathrm{C}$ in $5 \% \mathrm{CO}_{2}$. C2C12 cells were obtained from MilliporeSigma (catalog 91031101) and maintained in DMEM, 20\% FBS, and $50 \mu \mathrm{g} / \mathrm{ml}$ penicillin-streptomycin. Transfection was performed with Lipofectamine 2000 (Life Technologies, Thermo Fisher Scientific; catalog 11668019) according to the manufacturer's instructions. Twenty-four hours after transfection, cells were washed three times with ice-cold PBS. pDEST-mCherry-human p62 WT was a gift of Terje Johansen (University of Tromsø, The Arctic University of Norway, Tromsø, Norway) (46). pDEST-mCherry-human p62 P392L, M404V, and A390X were generated by mutagenesis in-house. GFP-human TIA1 WT (isoform A) has been previously reported, and GFP-human TIA1 E384K and N357S were generated by mutagenesis (7).

Immunocytochemistry and fluorescence microscopy. Cells were grown on glass coverslips prior to transfection with plasmid constructs. Twenty-four hours after transfection, cells were washed three times with PBS, fixed in $4 \%$ PFA for ten minutes, and permeabilized with $0.05 \%$ Triton $\mathrm{X}-100$ in PBS for ten minutes. After washing 3 times with PBS, cells were blocked with $2 \%$ BSA in PBS for 30 minutes to 1 hour at room temperature (RT). Cells were stained with a primary antibody at $4^{\circ} \mathrm{C}$ overnight followed by washing 3 times with PBS. Cells were incubated with Alexa 555- or Alexa 488 Fluor-conjugated secondary antibody at RT for 1 hour and mounted with Mowiol media containing DAPI. Images of 10 random fields were taken with a $\times 20$ objective using a Nikon Eclipse 80i fluorescence microscope. A blue channel was separated and used to count the total number of DAPIstained cells in Figure 3B, Figure 5B, and Supplemental Figure 3B. Cells costained with both TIA1 (green) and G3BP1 (red) were counted in a merge channel. For Figure 2, C and D, and Figure 6A, individual cells shown in a green or red channel were considered GFP-TIA1- or mCherry-SQSTM1-expressing cells and counted as a total. For Figure 6D, C2C12 cells expressing both mCherry-SQSTM1 and GFP-TIA1 were counted as a total in each red or green channel, while $\mathrm{C} 2 \mathrm{C} 12$ cells containing GFP-TIA1 puncta were counted in a merge channel. In Figure 3, C and D, individual SGs stained with TIA1 (green) were counted in a green channel as a total, while TIA1 SGs colocalized with SQSTM1 (red) were counted in a merge channel. For Figure 4, A and B, and Figure 4, C and D, individual endogenous TIA1 or GFP-TIA1 SGs (green) were counted as a total in a green channel, while TIA1 SGs colocalized with DRiPs (red) were counted in a merge channel. For Figure 5, A and $\mathrm{B}$, individual TIA1 puncta (green) were counted as a total in a green channel, and TIA1/TDP-43-positive puncta were counted in a merge channel. Cells or individual SGs were counted using ImageJ software (NIH). Frozen muscle biopsy sections ( $8-\mu \mathrm{m}$ thickness) were prepared on slides, fixed in 4\% PFA for 15 minutes, and immunostained using a protocol similar to that for cultured cells.

HS, inhibitor treatment, and DRiP labeling. MEFs or $\mathrm{C} 2 \mathrm{C} 12$ cells were incubated at $42^{\circ} \mathrm{C}$ with $5 \% \mathrm{CO}_{2}$ for 1 hour and returned to $37^{\circ} \mathrm{C}$ for the indicated durations (Figure 2, C and D; Figure 3, A and B; and Figures 4-6). For AsIII (MilliporeSigma; catalog 38150) treatment, control MEFs were treated with $0.5 \mathrm{mM}$ AsIII for 1 hour, and AsIII-containing media were replaced at the indicated time point (Figure $2 \mathrm{E}$ legend). To label DRiPs, control or $p 62^{-/-}$cells were incubated at $42^{\circ} \mathrm{C}$ with $5 \%$ $\mathrm{CO}_{2}$ for 1 hour and simultaneously treated with $25 \mu \mathrm{M}$ OP-puromycin for 30 minutes. For recovery experiments, cells were returned to $37^{\circ} \mathrm{C}$, and OP-puromycin-containing media were replaced for the indicated time (Figure 4 legend). Fixation and Click-iT reactions were performed with Alexa Azide 594 according to the manufacturer's instructions (Life Technologies, Thermo Fisher Scientific; catalog C10399). MG132 (C2211) and BAFA (B1793) were purchased from MilliporeSigma. Control MEFs and $p 62^{---}$were treated with MG132 $(20 \mu \mathrm{M})$ or BAFA (200 $\mathrm{nM}$ ) for 1 hour at $37^{\circ} \mathrm{C}$ with $5 \% \mathrm{CO}_{2}$ after 1 hour of $\mathrm{HS}$ at $42^{\circ} \mathrm{C}$.

Fluorescence recovery after photobleaching. Control MEFs were grown on 1.5 glass-bottomed 35-mm dishes (MatTek; P35G-1.5-10-C) and transfected with GFP-human TIA1 for 24 hours prior to imaging. Immediately before imaging, the medium was replaced with phenol red-free medium (Gibco, Thermo Fisher Scientific; catalog 21063029) containing 10\% FBS, $50 \mu \mathrm{g} / \mathrm{ml}$ penicillin-streptomycin, $1 \%$ sodium pyruvate, and $1 \%$ nonessential amino acid. The live-cell samples were treated with $0.5 \mathrm{mM}$ AsIII for 1 hour and placed on a heated chamber at $37^{\circ} \mathrm{C}$ with $5 \% \mathrm{CO}_{2}$. Imaging and photobleaching were performed with a $\times 40$ oil objective using a Nikon A1Rsi confocal microscope. Before bleaching, the images of prebleached TIA1 aggregates were taken. A 488-nm laser was used to photobleach TIA1 aggregates for $500 \mathrm{~ms}$. Immediately after bleaching, the images were collected every second for a total of 150 seconds as a post-bleached sample. The fluorescence intensities of post-bleached TIA1 aggregates (Ft) were individually measured. In the meantime, the fluorescence intensities of nonbleached TIA1 aggregates were measured as a reference control (Fref). Also, the fluorescence intensities of nonbleached and nontransfected background were measured as a background control (Fb). The photobleaching rate $(r)$ was calculated by comparing the fluorescence of the reference before (FrefO) and after (Fref) photobleaching: $\mathrm{r}=$ Fref/FrefO. The average of TIA1 aggregates fluorescence intensities ( $\mathrm{F}$ ) in different cells ( $n \geq 5$ cells containing TIA1 aggregates) was calculated as follows: $\mathrm{F}=(\mathrm{Ft}-\mathrm{Fb}) / \mathrm{r}$.

LDH assay. C2C12 cells were transfected with Cherry-p62-WT or AX along with GFP-TIA1-WT, -NS, or -EK 24 hours before the LDH assay. Cells were split into 96 -well plates with $2 \times 10^{5}$ cells per well, in triplicate. Cells were then incubated for 1 hour at $42^{\circ} \mathrm{C}$ in $5 \% \mathrm{CO}_{2}$ and returned to $37^{\circ} \mathrm{C}$ in $5 \% \mathrm{CO}_{2}$ for a 1 -hour recovery. The LDH assay was performed according to the manufacturer's instructions (Roche; catalog 04744926 001).

Statistics. A 2-tailed Student's $t$ test between 2 groups and 2-way ANOVA between more than 2 groups were performed. For all tests, $P$ values of less than 0.05 were considered statistically significant. Data are presented as the mean \pm SEM.

Study approval. All evaluations and genetic studies were conducted with the approval of the Human Studies Committee of Washington University and the IRB of Helsinki University. Written informed consent was provided by all study participants, in accordance with Declaration of Helsinki principles.

\section{Author contributions}

YJL designed and performed experiments, analyzed results, and wrote the manuscript. CCW conceived and designed experiments, analyzed results, and wrote the manuscript. BU conceived and designed experiments, analyzed results, and wrote the manuscript. JPT designed experiments, analyzed results, and provided feedback on the manuscript. PHJ, JS, JP, MS, and AV designed and performed experiments, analyzed results, and provided feedback on the manuscript. AE, TS, SP, MS, MJ, and PH designed studies and analyzed 
results. SHR and AA designed experiments, analyzed results, and provided key reagents. MCM, DHJ, PM, PC, JR, CK, TK, SZ, CS, and $H G$ provided key reagents and feedback on the manuscript.

\section{Acknowledgments}

CCW was supported by NIH (AG031867, AG042095, and AR068797); the Muscular Dystrophy Association; the Myositis Association; and the Hope Center for Neurological Disorders. BU was supported by the Academy of Finland; the Erkko Foundation; the Sigrid Jusélius Foundation; the Folkhälsan Institute of Genet- ics; and Tampere University Hospital Medical Research Funds. JPT was supported by the NIH (R35NS097974); St. Jude and the American Lebanese Syrian Associated Charities (ALSAC); and the Howard Hughes Medical Institute. We thank Helena Luque (the Folkhälsan Institute of Genetics) for technical assistance.

Address correspondence to: Conrad C. Weihl, Department of Neurology, Hope Center for Neurological Diseases, Washington University School of Medicine, St. Louis, Missouri 63110, USA. Phone: 314.362.6981; Email: weihlc@wustl.edu.
1. Bucelli RC, et al. SQSTM1 splice site mutation in distal myopathy with rimmed vacuoles. Neurology. 2015;85(8):665-674.

2. Watts GD, et al. Inclusion body myopathy associated with Paget disease of bone and frontotemporal dementia is caused by mutant valosin-containing protein. Nat Genet. 2004;36(4):377-381.

3. Kim HJ, et al. Mutations in prion-like domains in hnRNPA2B1 and hnRNPA1 cause multisystem proteinopathy and ALS. Nature. 2013;495(7442):467-473.

4. Taylor JP. Multisystem proteinopathy: intersecting genetics in muscle, bone, and brain degeneration. Neurology. 2015;85(8):658-660.

5. Johnson JO, et al. Mutations in the Matrin 3 gene cause familial amyotrophic lateral sclerosis. Nat Neurosci. 2014;17(5):664-666.

6. Hackman P, et al. Welander distal myopathy is caused by a mutation in the RNA-binding protein TIA1. Ann Neurol. 2013;73(4):500-509.

7. Mackenzie IR, et al. TIA1 mutations in amyotrophic lateral sclerosis and frontotemporal dementia promote phase separation and alter stress granule dynamics. Neuron. 2017;95(4):808-816.e9.

8. Deng HX, et al. Mutations in UBQLN2 cause dominant X-linked juvenile and adult-onset ALS and ALS/dementia. Nature. 2011;477(7363):211-215.

9. Maruyama $\mathrm{H}$, et al. Mutations of optineurin in amyotrophic lateral sclerosis. Nature. 2010;465(7295):223-226.

10. Lee Y, Chou TF, Pittman SK, Keith AL, Razani B, Weihl CC. Keap1/Cullin3 Modulates p62/ SQSTM1 Activity via UBA Domain Ubiquitination. Cell Rep. 2017;19(1):188-202.

11. Rea SL, Majcher V, Searle MS, Layfield R. SQSTM1 mutations--bridging Paget disease of bone and ALS/FTLD. Exp Cell Res. 2014;325(1):27-37.

12. Hocking LJ, et al. Domain-specific mutations in sequestosome 1 (SQSTM1) cause familial and sporadic Paget's disease. Hum Mol Genet. 2002;11(22):2735-2739.

13. Fecto F, et al. SQSTM1 mutations in familial and sporadic amyotrophic lateral sclerosis. Arch Neurol. 2011;68(11):1440-1446.

14. Le Ber I, et al. SQSTM1 mutations in French patients with frontotemporal dementia or frontotemporal dementia with amyotrophic lateral sclerosis. JAMA Neurol. 2013;70(11):1403-1410.

15. Evilä A, Arumilli M, Udd B, Hackman P. Targeted next-generation sequencing assay for detection of mutations in primary myopathies. Neuromuscul Disord. 2016;26(1):7-15.
16. Morissette J, Laurin N, Brown JP. Sequestosome 1: mutation frequencies, haplotypes, and phenotypes in familial Paget's disease of bone. J Bone Miner Res. 2006;21 Suppl 2:P38-P44.

17. Vieira NM, et al. A defect in the RNA-processing protein HNRPDL causes limb-girdle muscular dystrophy 1G (LGMD1G). Hum Mol Genet. 2014;23(15):4103-4110.

18. Gitcho MA, et al. TDP-43 A315T mutation in familial motor neuron disease. Ann Neurol. 2008;63(4):535-538.

19. Kwiatkowski TJ, et al. Mutations in the FUS/ TLS gene on chromosome 16 cause familial amyotrophic lateral sclerosis. Science. 2009;323(5918):1205-1208.

20. Molliex A, et al. Phase separation by low complexity domains promotes stress granule assembly and drives pathological fibrillization. Cell. 2015;163(1):123-133.

21. Powell A, Baker-Austin C, Wagley S, Bayley A, Hartnell R. Isolation of pandemic Vibrio parahaemolyticus from UK water and shellfish produce. Microb Ecol. 2013;65(4):924-927.

22. Ju JS, Weihl CC. Inclusion body myopathy, Paget's disease of the bone and fronto-temporal dementia: a disorder of autophagy. Hum Mol Genet. 2010;19(R1):R38-R45.

23. Weihl CC, et al. TDP-43 accumulation in inclusion body myopathy muscle suggests a common pathogenic mechanism with frontotemporal dementia. J Neurol Neurosurg Psychiatry. 2008;79(10):1186-1189.

24. Salajegheh M, et al. Sarcoplasmic redistribution of nuclear TDP-43 in inclusion body myositis. Muscle Nerve. 2009;40(1):19-31.

25. Nogalska A, Terracciano C, D’Agostino C, King Engel W, Askanas V. p62/SQSTM1 is overexpressed and prominently accumulated in inclusions of sporadic inclusion-body myositis muscle fibers, and can help differentiating it from polymyositis and dermatomyositis. Acta Neuropathol. 2009;118(3):407-413.

26. Hiniker A, Daniels BH, Lee HS, Margeta M. Comparative utility of LC3, p62 and TDP-43 immunohistochemistry in differentiation of inclusion body myositis from polymyositis and related inflammatory myopathies. Acta Neuropathol Commun. 2013;1:29.

27. Buchan JR, Kolaitis RM, Taylor JP, Parker R. Eukaryotic stress granules are cleared by autophagy and Cdc48/VCP function. Cell. 2013;153(7):1461-1474.

28. Seguin SJ, et al. Inhibition of autophagy, lysosome and VCP function impairs stress granule assem- bly. Cell Death Differ. 2014;21(12):1838-1851.

29. Falchetti A, et al. Two novel mutations at exon 8 of the Sequestosome 1 (SQSTM1) gene in an Italian series of patients affected by Paget's disease of bone (PDB). J Bone Miner Res. 2004;19(6):1013-1017.

30. Cuyvers E, et al. Genetic variability in SQSTM1 and risk of early-onset Alzheimer dementia: a European early-onset dementia consortium study. Neurobiol Aging. 2015;36(5):2005.e15-2005.e22.

31. Langston AL, et al. Randomized trial of intensive bisphosphonate treatment versus symptomatic management in Paget's disease of bone. J Bone Miner Res. 2010;25(1):20-31.

32. Ganassi M, et al. A surveillance function of the HSPB8-BAG3-HSP70 chaperone complex ensures stress granule integrity and dynamism. Mol Cell. 2016;63(5):796-810.

33. Meriin AB, Zaarur N, Sherman MY. Association of translation factor eEF1A with defective ribosomal products generates a signal for aggresome formation. J Cell Sci. 2012;125(Pt 11):2665-2674.

34. Schubert U, Antón LC, Gibbs J, Norbury CC, Yewdell JW, Bennink JR. Rapid degradation of a large fraction of newly synthesized proteins by proteasomes. Nature. 2000;404(6779):770-774.

35. Teyssou E, et al. Mutations in SQSTM1 encoding p62 in amyotrophic lateral sclerosis: genetics and neuropathology. Acta Neuropathol. 2013;125(4):511-522.

36. Welander L. Homozygous appearance of distal myopathy. Acta Genet Stat Med.1957;7(2):321-325.

37. Al-Obeidi E, et al. Genotype-phenotype study in patients with valosin-containing protein mutations associated with multisystem proteinopathy. Clin Genet. 2018;93(1):119-125.

38. Mehta SG, et al. APOE is a potential modifier gene in an autosomal dominant form of frontotemporal dementia (IBMPFD). Genet Med. 2007;9(1):9-13.

39. Weihl CC, et al. Targeted sequencing and identification of genetic variants in sporadic inclusion body myositis. Neuromuscul Disord. 2015;25(4):289-296.

40. Gang Q, et al. Rare variants in SQSTM1 and VCP genes and risk of sporadic inclusion body myositis. Neurobiol Aging. 2016;47:218.e1-218.e9.

41. Abramzon Y, et al. Valosin-containing protein (VCP) mutations in sporadic amyotrophic lateral sclerosis. Neurobiol Aging. 2012;33(9):2231.e1-2231.e6.

42. Lucas GJ, et al. Evaluation of the role of Valosin-containing protein in the pathogenesis of familial and sporadic Paget's disease of bone. Bone. 2006;38(2):280-285. 
43. Beyens G, et al. Evaluation of the role of the SQSTM1 gene in sporadic Belgian patients with Paget's disease. Calcif Tissue Int. 2004;75(2):144-152.

44. Lemmers RJ, et al. Digenic inheritance of an SMCHD1 mutation and an FSHD-permissive D4Z4 allele causes facioscapulohumeral muscular dystrophy type 2. Nat Genet. 2012;44(12):1370-1374 .

45. Kedersha NL, Gupta M, Li W, Miller I, Anderson P. RNA-binding proteins TIA-1 and TIAR link the phosphorylation of eIF-2 alpha to the assembly of mammalian stress granules. JCell Biol.
1999;147(7):1431-1442.

46. Pankiv S, et al. p62/SQSTM1 binds directly to Atg8/LC3 to facilitate degradation of ubiquitinated protein aggregates by autophagy. J Biol Chem. 2007;282(33):24131-24145.

47. Mateju D, et al. An aberrant phase transition of stress granules triggered by misfolded protein and prevented by chaperone function. $E M B O J$. 2017;36(12):1669-1687.

48. Ratti A, Buratti E. Physiological functions and pathobiology of TDP-43 and FUS/TLS proteins.
J Neurochem. 2016;138 Suppl 1:95-111.

49. Ju JS, et al. Valosin-containing protein (VCP) is required for autophagy and is disrupted in VCP disease. JCell Biol. 2009;187(6):875-888.

50. Scotter EL, et al. Differential roles of the ubiquitin proteasome system and autophagy in the clearance of soluble and aggregated TDP-43 species. J Cell Sci. 2014;127(Pt 6):1263-1278.

51. Lek M, et al. Analysis of protein-coding genetic variation in 60,706 humans. Nature. 2016;536(7616):285-291. 